\title{
Star-shaped Mesogens of Triazine-Based Dendrons and Dendrimers as Unconventional Columnar Liquid Crystals
}

Long-Li Lai, ${ }^{*}$ a Cheng-Hua Lee, ${ }^{a}$ Ling-Yung Wang, ${ }^{a}$ Kung-Lung Cheng ${ }^{b}$ and Hsiu-Fu $\mathrm{Hsu}^{\mathrm{c}}$

${ }^{a}$ Department of Applied Chemistry, National Chi Nan University, Puli, Nantou, Taiwan 545. E-mail: lilai@ncnu.edu.tw

${ }^{\mathrm{b}}$ Applied Chemistry Division, Material and Chemical Research Laboratories, Industrial Research Institute, Hsinchu, Taiwan 300.

${ }^{\mathbf{c}}$ Department of Chemistry, Tamkang University, Tamsui, Taiwan 251.

Contents:

${ }^{1} \mathrm{H}$ NMR of $\mathbf{G}_{\mathbf{2}}-\mathbf{N} \sim \mathbf{N}-\mathbf{G}_{\mathbf{2}}(\mathrm{R}=$ dioctylamine $) \quad \mathrm{S} 2$

${ }^{13} \mathrm{C}$ NMR of $\mathbf{G}_{\mathbf{2}}-\mathbf{N} \sim \mathbf{N}-\mathbf{G}_{\mathbf{2}}(\mathrm{R}=$ dioctylamine) $\mathrm{S} 3$

${ }^{1} \mathrm{H}$ NMR of $\mathbf{G}_{\mathbf{3}}-\mathbf{N} \sim \mathbf{N}-\mathbf{G}_{\mathbf{3}}(\mathrm{R}=$ dioctylamine $) \quad \mathrm{S} 4$

${ }^{13} \mathrm{C}$ NMR of $\mathbf{G}_{\mathbf{3}}-\mathbf{N} \sim \mathbf{N}-\mathbf{G}_{3}$ (R=dioctylamine) S5

${ }^{1} \mathrm{H}$ NMR of $\mathbf{G}_{\mathbf{4}}-\mathbf{N} \sim \mathbf{N}-\mathbf{G}_{\mathbf{4}} \quad(\mathrm{R}=$ dioctylamine $) \quad \mathrm{S} 6$

${ }^{13} \mathrm{C}$ NMR of $\mathbf{G}_{\mathbf{4}}-\mathbf{N} \sim \mathbf{N}-\mathbf{G}_{\mathbf{4}}(\mathrm{R}=$ dioctylamine $) \quad \mathrm{S} 7$

General characterization methods $\quad \mathrm{S} 8$

General computational data S9

Parameters of $\mathbf{G}_{\mathbf{4}}-\mathbf{C l}$ (R=piperidine) S9

Parameters of $\mathbf{G}_{\mathbf{4}}-\mathbf{N H}$ (R=piperidine) $\quad$ S24

Parameters of $\mathbf{G}_{\mathbf{4}}-\mathbf{N} \sim \mathbf{N}-\mathbf{G}_{\mathbf{4}}(\mathrm{R}=$ piperidine $) \quad$ S39 
${ }^{1} \mathrm{H}$ NMR of $\mathbf{G}_{\mathbf{2}}-\mathbf{N} \sim \mathbf{N}-\mathbf{G}_{\mathbf{2}}(\mathrm{R}=$ dioctylamine $)$
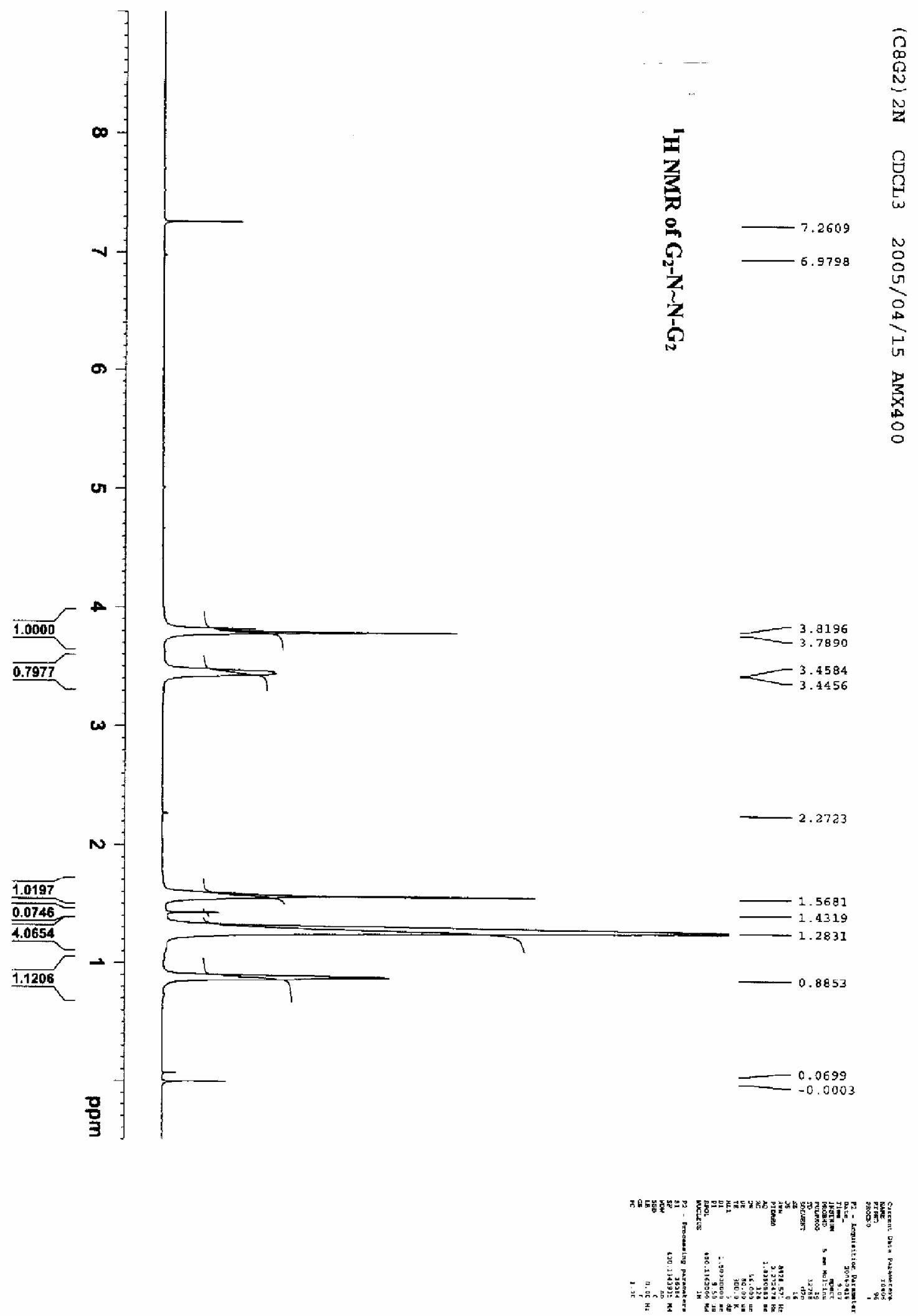
${ }^{13} \mathrm{C}$ NMR of $\mathbf{G}_{\mathbf{2}}-\mathbf{N} \sim \mathbf{N}-\mathbf{G}_{\mathbf{2}}(\mathrm{R}=$ dioctylamine $)$

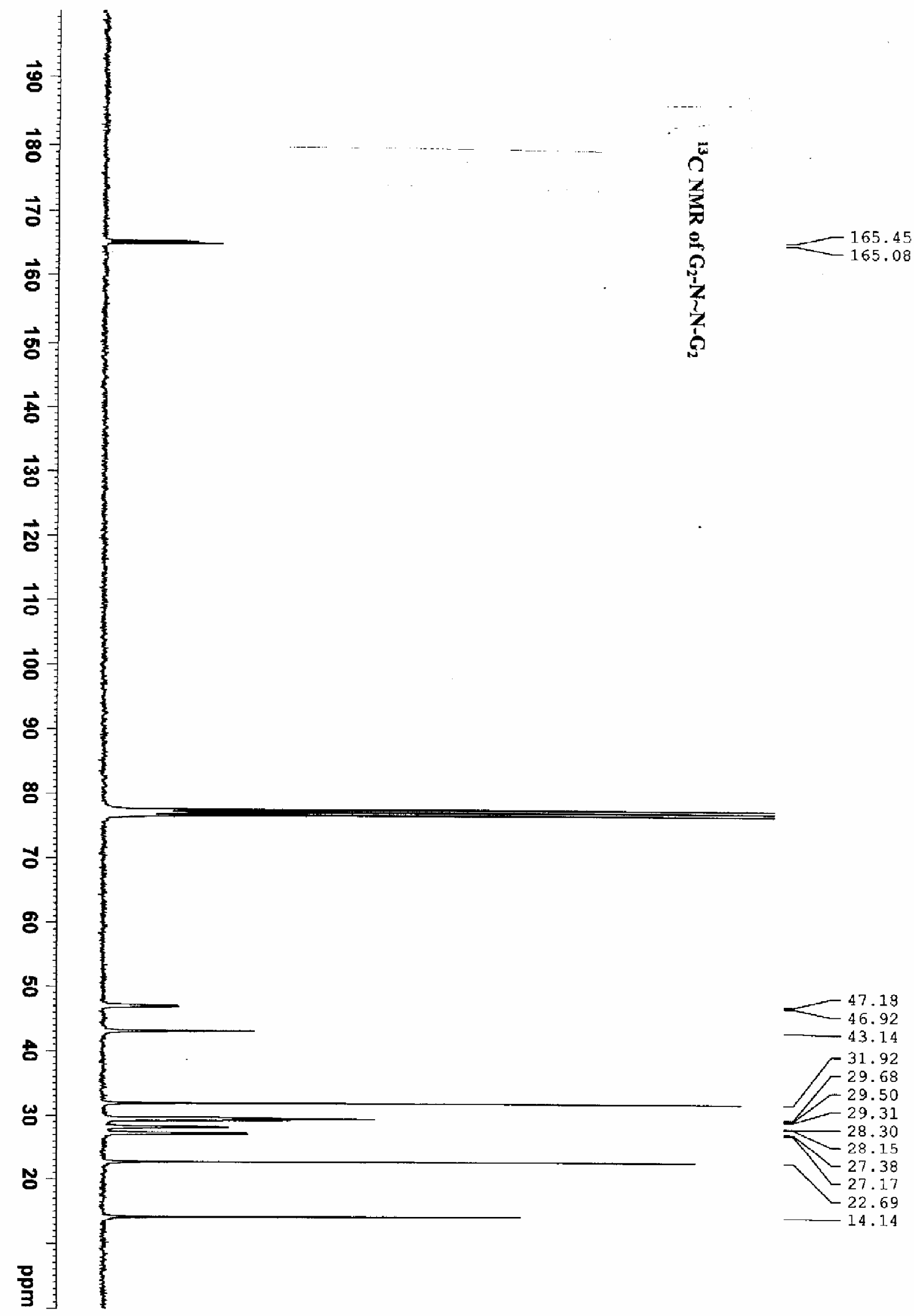


${ }^{1} \mathrm{H}$ NMR of $\mathbf{G}_{\mathbf{3}}-\mathbf{N} \sim \mathbf{N}-\mathbf{G}_{\mathbf{3}}(\mathrm{R}=$ dioctylamine $)$

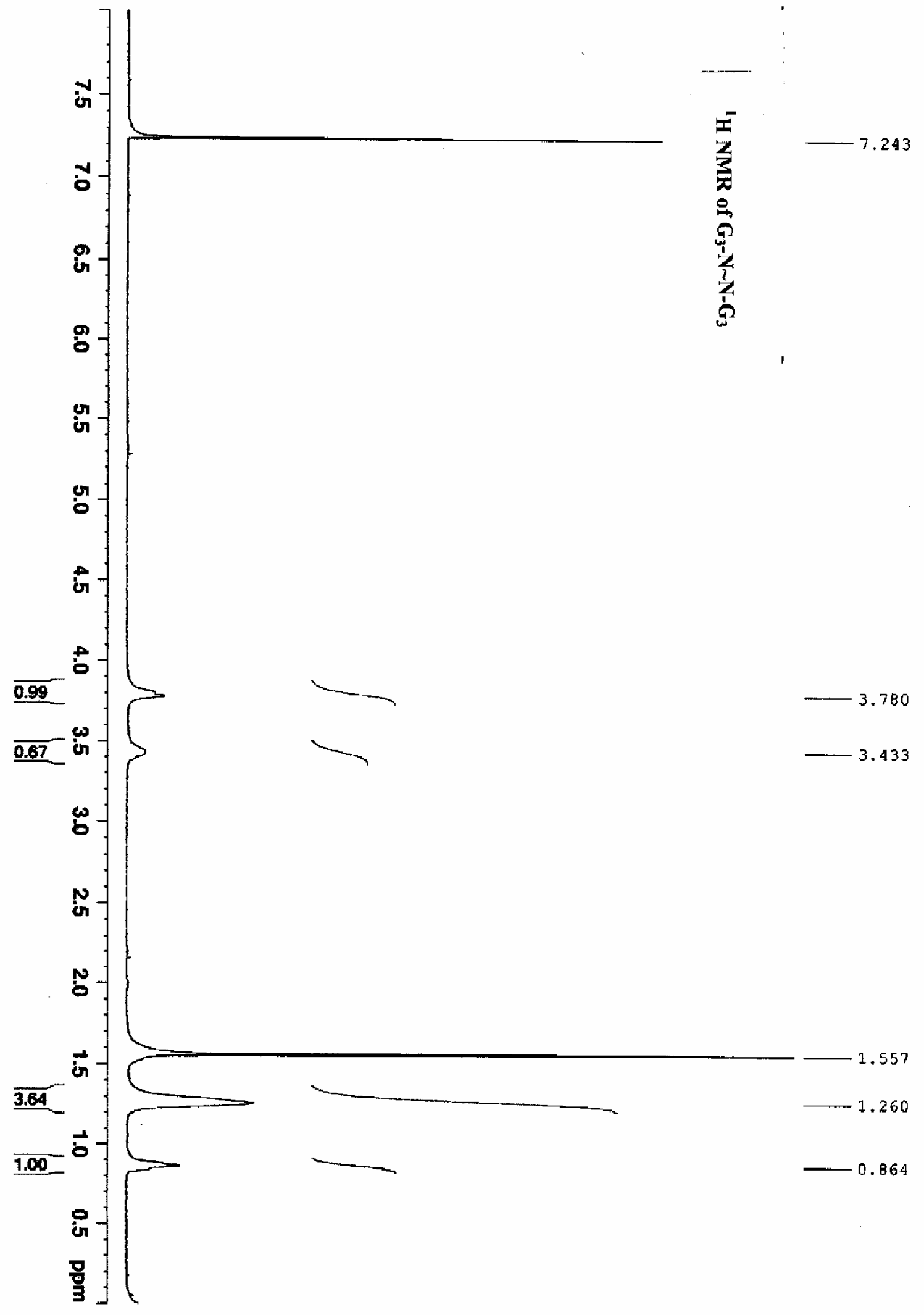




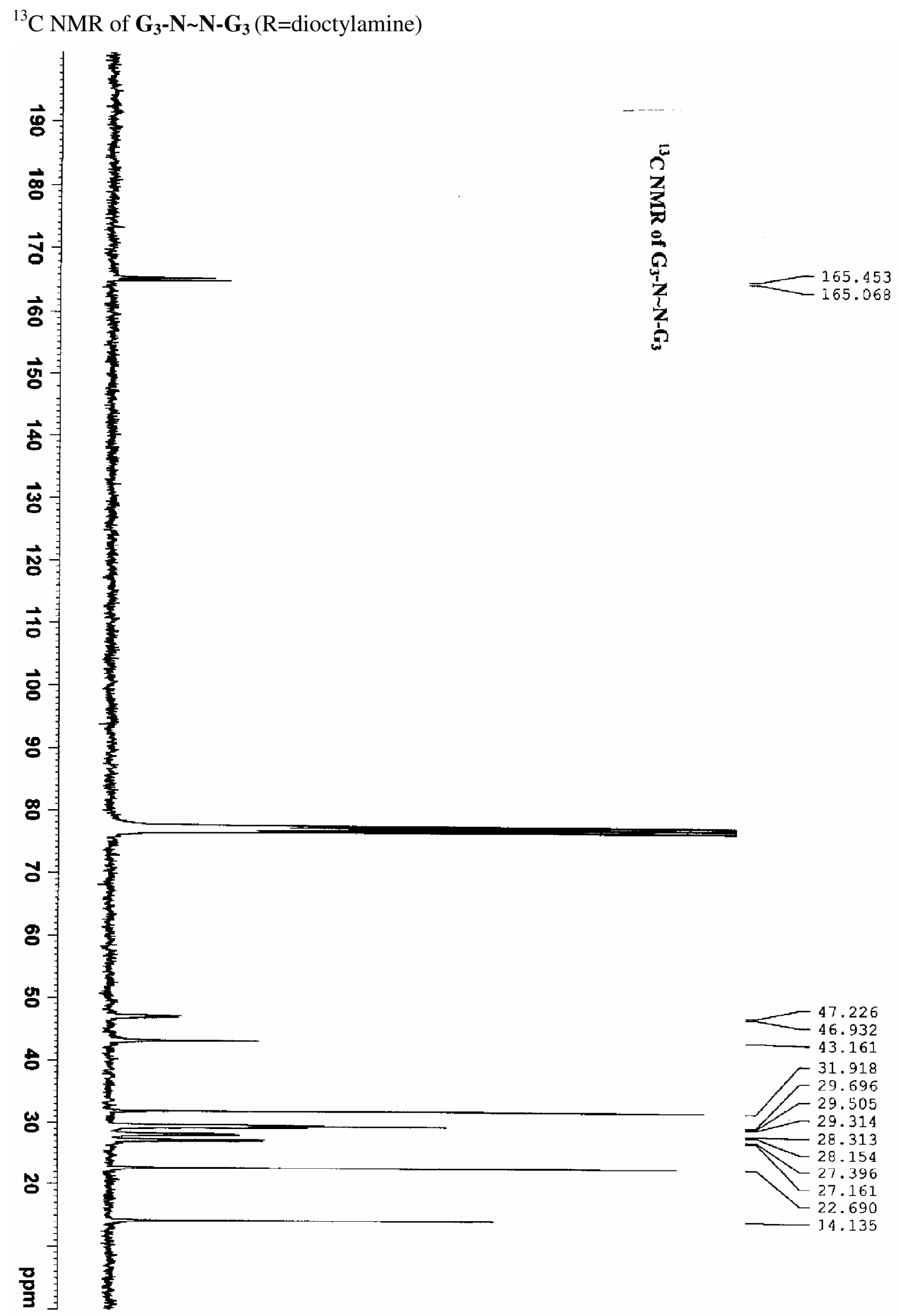


${ }^{1} \mathrm{H}$ NMR of $\mathbf{G}_{\mathbf{4}}-\mathbf{N} \sim \mathbf{N}-\mathbf{G}_{\mathbf{4}}(\mathrm{R}=$ dioctylamine $)$
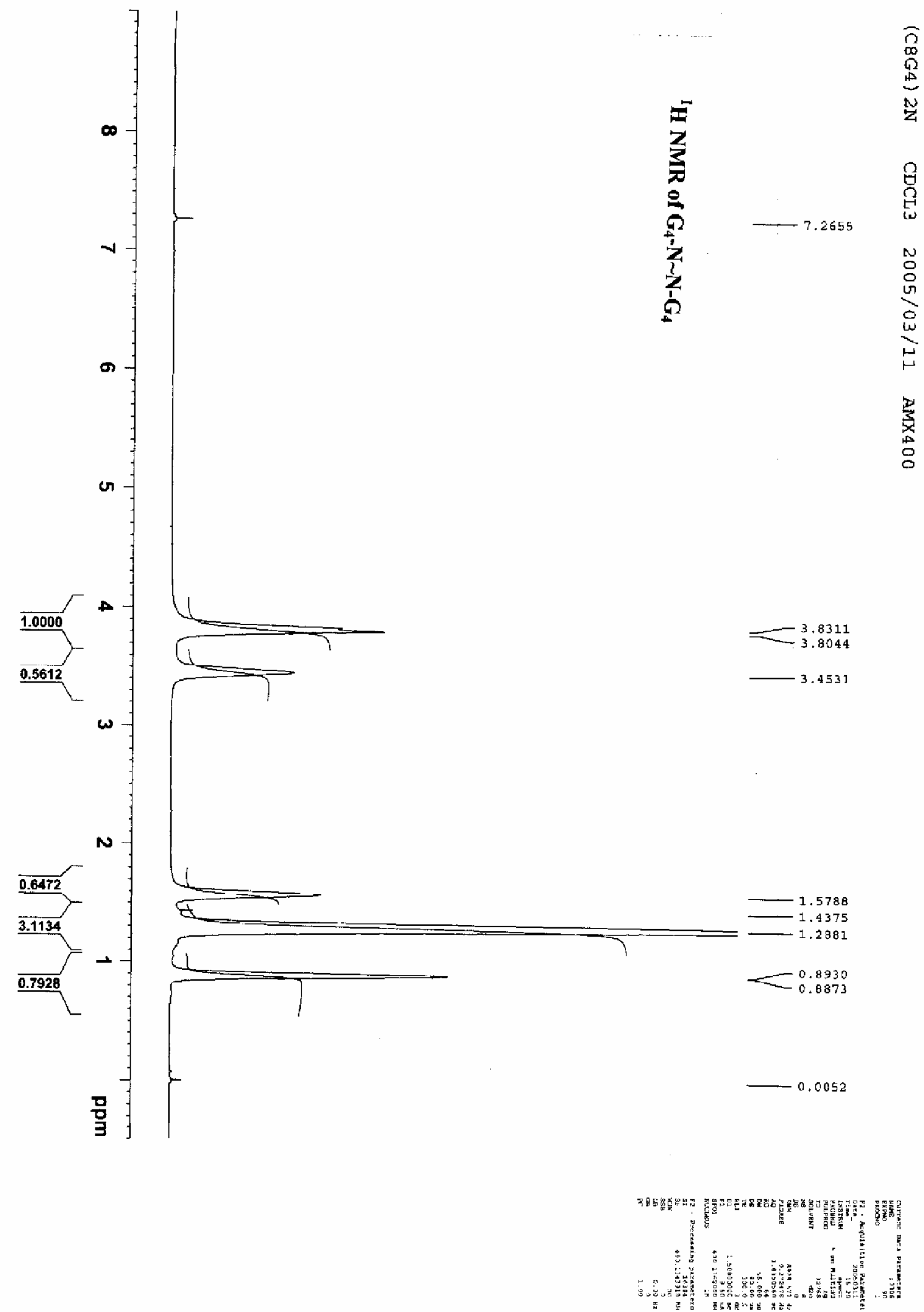
${ }^{13} \mathrm{C}$ NMR of $\mathbf{G}_{\mathbf{4}}-\mathbf{N} \sim \mathbf{N}-\mathbf{G}_{\mathbf{4}}(\mathrm{R}=$ dioctylamine $)$
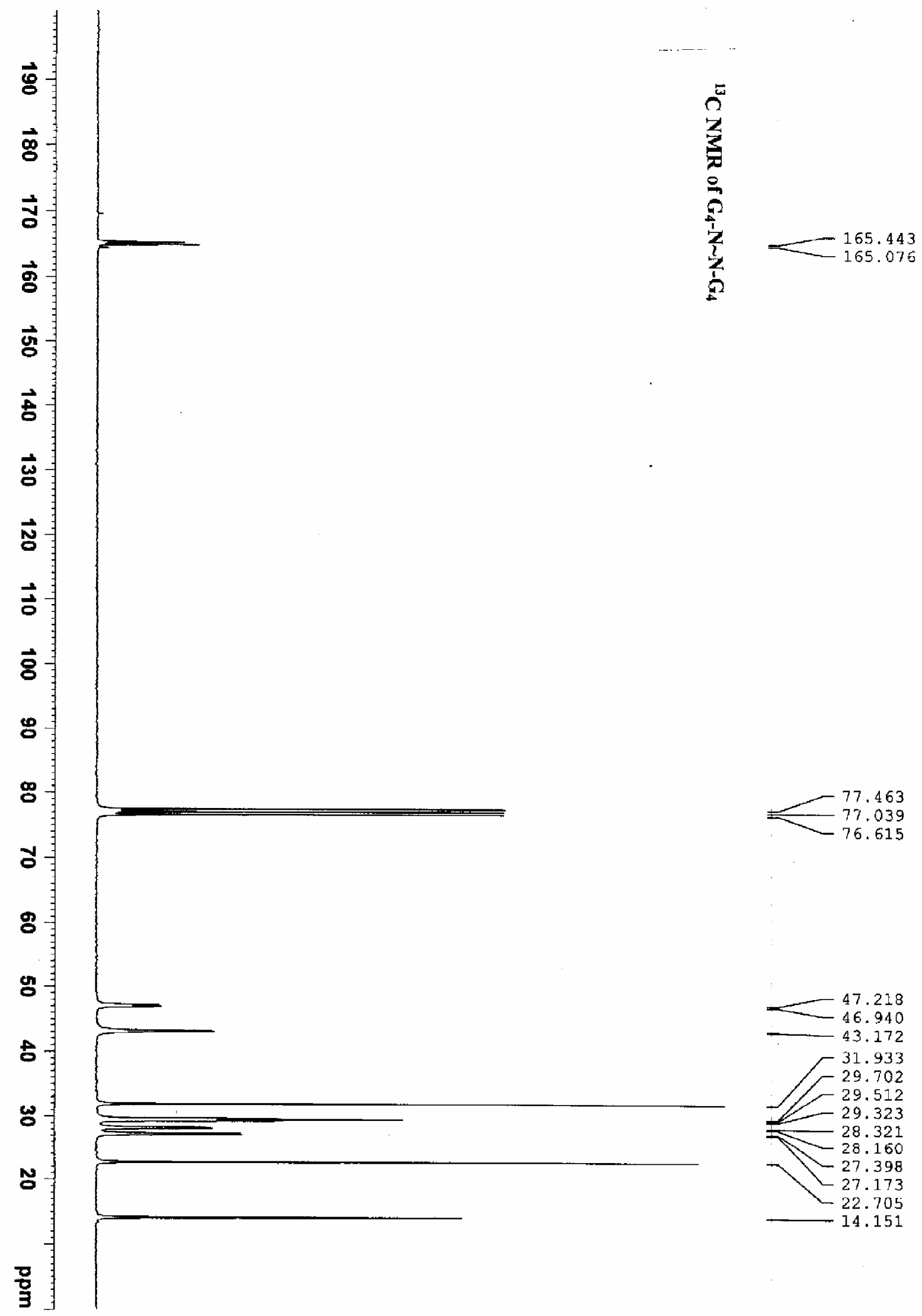

47.218

46.940

43.172

$-31.933$

29.702

$-29.512$

29.323

28.321

$-28.160$

$-27.398$

$-27.173$

$-22.705$

$-14.151$ 
General characterization methods: ${ }^{1} \mathrm{H}-\mathrm{NMR}$ and ${ }^{13} \mathrm{C}-\mathrm{NMR}$ spectra in $\mathrm{CDCl}_{3}$ were recorded on a NMR spectrometer. High resolution mass spectra (HRMS) and mass spectra were obtained from a spectrometer (EI, 70ev). All MALDI-TOF mass spectra were performed on a time-of flight mass spectrometer. The mesogenic behaviors and phase transitions were characterized by polarizing optical microscopy and differential scanning calorimetry. The thermogravimetric behaviour was analyzed by TGA under nitrogen. The XRD studies were carried out at the temperature ranges of mesophases. Synchrotron power X-ray diffraction (XRD) measurements were performed at beam line BL17A of the National Synchrotron Radiation Research Center (NSRRC), Taiwan, where the X-ray wavelength is $1.334431 \AA$. The XRD data are collected using imaging plates (IP, an area $=20 \times 40 \mathrm{~cm}^{2}$ and a pixel resolution of 100) curved with a radius equivalent to the sample-to-image plate distance of $280 \mathrm{~mm}$, and the diffraction signals were accumulated for $3 \mathrm{~min}$. The powder samples were packed into a capillary tube and heated by a heat gun, where the temperature controller is programmable by a PC with a PID feed back system. The scattering angle theta was calibrated by a mixture of silver and silicon. The UV absorbance of the dendrimer was recorded from a UV-vis spectrometer. 
General computational data: The simulation of $\mathbf{G}_{\mathbf{4}}-\mathbf{C l}, \mathbf{G}_{\mathbf{4}}-\mathbf{N H}$ and $\mathbf{G}_{\mathbf{4}}-\mathbf{N} \sim \mathbf{N G}_{\mathbf{4}}$ ( $\mathrm{R}=$ piperidine) was carried out in CAChe program using MM2 theory which is a traditional mechanic force field. Therefore no basis set is needed for the calculation and no frequencies were obtained. The energy of the optimized $\mathbf{G}_{\mathbf{4}}-\mathbf{C l}, \mathbf{G}_{\mathbf{4}}-\mathbf{N H}$ and $\mathbf{G}_{\mathbf{4}} \mathbf{- N} \sim \mathbf{N G}_{\mathbf{4}}$ are $54.37,62.12$ and $28.80 \mathrm{kcal} / \mathrm{mol}$.

$$
\begin{array}{llll}
\text { Parameters of } & \mathbf{G}_{4}-\mathbf{C l}(\mathrm{R}=\text { piperidine }) \\
\mathrm{C} & 13.5245 & -20.1029 & 4.628 \\
\mathrm{C} & 14.9534 & -19.5621 & 4.4641 \\
\mathrm{~N} & 12.066 & -15.5843 & 9.4812 \\
\mathrm{H} & 14.4528 & -17.4607 & 4.7314 \\
\mathrm{~N} & 13.4263 & -18.9734 & 6.8154 \\
\mathrm{H} & 7.3573 & -20.9951 & 6.4294 \\
\mathrm{C} & 6.4938 & -19.4484 & 7.7161 \\
\mathrm{~N} & 12.1662 & -12.7859 & 9.7013 \\
\mathrm{C} & 15.1238 & -18.222 & 5.1965 \\
\mathrm{C} & 11.0293 & -14.9115 & 10.3057 \\
\mathrm{H} & 11.4032 & -14.922 & 11.3564 \\
\mathrm{H} & 10.049 & -15.4403 & 10.2953 \\
\mathrm{C} & 10.8463 & -13.4557 & 9.8416 \\
\mathrm{H} & 10.1564 & -12.9226 & 10.5352 \\
\mathrm{H} & 10.3783 & -13.4474 & 8.8293 \\
\mathrm{C} & 8.9067 & -20.2069 & 7.7547 \\
\mathrm{C} & 7.6541 & -20.0208 & 6.8869 \\
\mathrm{C} & 13.2094 & -13.4683 & 8.8911 \\
\mathrm{H} & 12.8453 & -13.4659 & 7.8369 \\
\mathrm{H} & 14.1913 & -12.9426 & 8.909 \\
\mathrm{C} & 13.3902 & -14.9208 & 9.3666 \\
\mathrm{H} & 14.093 & -15.4579 & 8.6895 \\
\mathrm{C} & 22.9451 & -8.8201 & 6.404 \\
\mathrm{C} & 8.1399 & -18.3025 & 9.2568 \\
\mathrm{C} & 6.8914 & -18.1217 & 8.382 \\
\mathrm{H} & 13.8392 & -14.9257 & 10.3874 \\
\mathrm{C} & 20.7886 & -2.5503 & 10.3763 \\
\mathrm{C} & 19.7694 & -1.8473 & 11.2866 \\
\mathrm{~N} & 17.5902 & -8.4938 & 10.9436 \\
\mathrm{C} & 14.7702 & -18.363 & 6.6838 \\
\mathrm{H} & 15.4901 & -19.0519 & 7.1844 \\
& & &
\end{array}
$$




$\begin{array}{llll}\mathrm{H} & 14.8533 & -17.3722 & 7.186 \\ \mathrm{C} & 13.1633 & -20.2515 & 6.1126 \\ \mathrm{H} & 17.8311 & -2.23 & 12.2305 \\ \mathrm{H} & 12.1091 & -20.5978 & 6.2103 \\ \mathrm{~N} & 20.0178 & -4.7034 & 11.2884 \\ \mathrm{H} & 22.1766 & -6.7865 & 6.5148 \\ \mathrm{H} & 12.8067 & -19.3974 & 4.1453 \\ \mathrm{C} & 12.5267 & -18.419 & 7.5124 \\ \mathrm{~N} & 12.7647 & -17.2694 & 8.1476 \\ \mathrm{H} & 15.1876 & -19.4336 & 3.38 \\ \mathrm{C} & 11.8258 & -16.6754 & 8.8872 \\ \mathrm{~N} & 14.9381 & -9.294 & 10.4798 \\ \mathrm{~N} & 10.6271 & -17.2539 & 8.9843 \\ \mathrm{C} & 10.3555 & -18.4016 & 8.3594 \\ \mathrm{~N} & 9.2122 & -18.9364 & 8.454 \\ \mathrm{~N} & 11.3172 & -18.971 & 7.6297 \\ \mathrm{C} & 18.5483 & -2.7432 & 11.5461 \\ \mathrm{H} & 13.4225 & -21.0863 & 4.109 \\ \mathrm{C} & 17.3641 & -9.8586 & 10.403 \\ \mathrm{H} & 17.3375 & -10.548 & 11.2792 \\ \mathrm{H} & 18.1833 & -10.2038 & 9.7315 \\ \mathrm{C} & 16.0146 & -9.9142 & 9.6632 \\ \mathrm{H} & 15.7874 & -10.9663 & 9.3753 \\ \mathrm{H} & 16.0851 & -9.3127 & 8.7267 \\ \mathrm{C} & 22.8062 & -7.4107 & 8.4998 \\ \mathrm{C} & 22.9911 & -7.3947 & 6.976 \\ \mathrm{C} & 15.1835 & -7.9456 & 11.0575 \\ \mathrm{H} & 15.2228 & -7.2358 & 10.198 \\ \mathrm{H} & 14.3712 & -7.6045 & 11.7395 \\ \mathrm{C} & 16.5274 & -7.9145 & 11.8039 \\ \mathrm{H} & 16.7513 & -6.878 & 12.1446 \\ \mathrm{H} & 15.6811 & -20.304 & 4.871 \\ \mathrm{H} & 16.1716 & -17.8525 & 5.0856 \\ \mathrm{H} & 21.4381 & -9.5469 & 8.3016 \\ \mathrm{H} & -9.5237 & 6.7789 \\ \mathrm{H} & -4.0928 & 12.142\end{array}$




$\begin{array}{llll}\mathrm{H} & 19.4268 & -3.9448 & 13.1479 \\ \mathrm{H} & 18.0774 & -4.7424 & 12.2765 \\ \mathrm{C} & 21.2202 & -3.898 & 10.9717 \\ \mathrm{H} & 21.9208 & -4.4128 & 10.2753 \\ \mathrm{H} & 21.6809 & -1.8973 & 10.2215 \\ \mathrm{C} & 19.8844 & -5.8791 & 10.8387 \\ \mathrm{~N} & 18.7985 & -6.5979 & 11.13 \\ \mathrm{C} & 18.6329 & -7.8358 & 10.6602 \\ \mathrm{H} & 9.7491 & -20.5764 & 7.1266 \\ \mathrm{H} & 8.7171 & -20.9738 & 8.5417 \\ \mathrm{H} & 6.1988 & -20.1848 & 8.5011 \\ \mathrm{H} & 7.8892 & -19.3199 & 6.0505 \\ \mathrm{~N} & 19.5843 & -8.357 & 9.883 \\ \mathrm{C} & 20.6852 & -7.6667 & 9.5759 \\ \mathrm{~N} & 21.5821 & -8.1736 & 8.8403 \\ \mathrm{~N} & 20.8196 & -6.4304 & 10.0622 \\ \mathrm{H} & 20.3275 & -2.7247 & 9.3749 \\ \mathrm{H} & 19.4422 & -0.8877 & 10.819 \\ \mathrm{H} & 18.0139 & -2.9221 & 10.5826 \\ \mathrm{H} & 22.7956 & -6.3668 & 8.8885 \\ \mathrm{H} & 23.662 & -7.9377 & 8.9831 \\ \mathrm{H} & 20.2543 & -1.5863 & 12.2565 \\ \mathrm{H} & 23.9597 & -6.9052 & 6.7132 \\ \mathrm{H} & 20.458 & -10.0149 & 8.5492 \\ \mathrm{H} & 22.2332 & -10.1669 & 8.7779 \\ \mathrm{H} & 8.4396 & -17.3233 & 9.6951 \\ \mathrm{H} & 7.9169 & -18.9921 & 10.1043 \\ \mathrm{H} & 6.0459 & -17.7303 & 8.9973 \\ \mathrm{H} & 7.1053 & -17.3628 & 7.5918 \\ \mathrm{H} & 20.7766 & -8.9807 & 6.3096 \\ \mathrm{H} & 21.6239 & -10.5652 & 6.3763 \\ \mathrm{H} & 21.7636 & -3.7346 & 11.9319 \\ \mathrm{~N} & 12.3889 & -11.6625 & 10.2395 \\ \mathrm{~N} & 11.6735 & -9.8699 & 11.5295 \\ \mathrm{H} & -11.055 & 10.9613 \\ \mathrm{H} & -21.0229 & 6.5885\end{array}$




\begin{tabular}{|c|c|c|c|}
\hline & & & \\
\hline & 13.5724 & -11.0619 & \\
\hline & & & \\
\hline & & & \\
\hline & & & \\
\hline & & & \\
\hline & & & \\
\hline & & & \\
\hline & & & \\
\hline & & & \\
\hline & & & \\
\hline & & & \\
\hline & 8.872 & & \\
\hline & & & \\
\hline & & & \\
\hline & & & \\
\hline & 9.50 & & \\
\hline & & & \\
\hline & & & \\
\hline & 18.9 & & \\
\hline & & & \\
\hline & & & \\
\hline & & & \\
\hline & & -0.37 & \\
\hline & & & \\
\hline & & & \\
\hline & & & \\
\hline & 14.1641 & & \\
\hline & 13. & & \\
\hline & & & \\
\hline & & & \\
\hline & 12. & & \\
\hline & 477 & & 90 \\
\hline & 20.3205 & & 80 \\
\hline & & & \\
\hline & 12.5604 & & \\
\hline & & 2.9955 & \\
\hline & 11.8215 & 3.1823 & \\
\hline
\end{tabular}




\begin{tabular}{|c|c|c|c|}
\hline & & & \\
\hline & & 3.3726 & \\
\hline & 9307 & & \\
\hline & & & \\
\hline & & & \\
\hline & & & \\
\hline & & & \\
\hline & & & \\
\hline & 6.7 & 3.6 & \\
\hline & & 5.30 & \\
\hline & & & \\
\hline & & & \\
\hline & 19.6 & & \\
\hline & 0.3 & 78 & \\
\hline & & & \\
\hline & & & \\
\hline & 4.964 & & \\
\hline & & 5.452 & 101 \\
\hline & & 3.0 & \\
\hline & 16.4764 & 2.78 & \\
\hline & 19.0 & & \\
\hline & & 1.5 & 42 \\
\hline & 7.9346 & 2.31 & 069 \\
\hline & 16.7896 & 0.6248 & \\
\hline & 18. & 0.8 & \\
\hline & & & \\
\hline & 18.5661 & 2.0712 & \\
\hline & & & \\
\hline & 20 & 6.0 & \\
\hline & & 3.91 & 20.949 \\
\hline & 8.7911 & 3.2 & \\
\hline & 8.47 & & \\
\hline & 8.4468 & & \\
\hline & 9.531 & 3.7964 & 1004 \\
\hline & & & \\
\hline & 5.3975 & 8.7184 & \\
\hline & 5.4707 & 10.0367 & 23.003 \\
\hline & 6.5145 & 2.0061 & \\
\hline
\end{tabular}




\begin{tabular}{|c|c|c|c|}
\hline & & & \\
\hline & & .9603 & \\
\hline & & & \\
\hline & 5.1332 & & \\
\hline & 19.3868 & & \\
\hline & & & \\
\hline & & & \\
\hline & & & \\
\hline & & & \\
\hline & & & \\
\hline & & & \\
\hline & & & \\
\hline & & & \\
\hline & & & \\
\hline & & & \\
\hline & & & \\
\hline & & & \\
\hline & & & \\
\hline & & & \\
\hline & & & \\
\hline & 20.8 & & \\
\hline & 20.8764 & & \\
\hline & 20.4362 & 0.0965 & \\
\hline & & & \\
\hline & & & \\
\hline & & & \\
\hline & 4.4816 & & \\
\hline & & & \\
\hline & -0.9756 & & \\
\hline & & & \\
\hline & 4.3341 & 73 & 23.5 \\
\hline & 5.8174 & 583 & \\
\hline & & & \\
\hline & & & \\
\hline & & & \\
\hline & 8.167 & 8.1416 & 22700 \\
\hline & 17.2284 & -1.4955 & 10.1 \\
\hline & 18.6846 & -2.0569 & 17.6 \\
\hline
\end{tabular}




$\begin{array}{llll}\mathrm{H} & 18.5916 & -3.1311 & 15.3686 \\ \mathrm{H} & 18.459 & -1.5232 & 14.5741 \\ \mathrm{H} & 7.2673 & 9.1988 & 21.0244 \\ \mathrm{H} & 8.7726 & 9.6611 & 21.8925 \\ \mathrm{H} & 1.6184 & 5.3761 & 24.6676 \\ \mathrm{C} & 10.7925 & 0.8987 & 17.69 \\ \mathrm{~N} & 10.3068 & -0.2944 & 17.3477 \\ \mathrm{C} & 9.0314 & -0.6213 & 17.5649 \\ \mathrm{~N} & 5.9896 & -8.4314 & 17.3982 \\ \mathrm{~N} & 8.2267 & 0.2628 & 18.159 \\ \mathrm{H} & 20.1079 & 4.7319 & 19.0347 \\ \mathrm{C} & 8.6874 & 1.4678 & 18.5093 \\ \mathrm{~N} & 9.9642 & 1.7735 & 18.2621 \\ \mathrm{H} & 6.964 & 11.3999 & 22.1762 \\ \mathrm{H} & 7.419 & 10.6673 & 23.751 \\ \mathrm{~N} & 8.6173 & -1.7556 & 17.188 \\ \mathrm{~N} & 7.7804 & -4.379 & 16.3614 \\ \mathrm{C} & 7.2144 & -2.18 & 17.4324 \\ \mathrm{H} & 6.6137 & -1.8015 & 16.5721 \\ \mathrm{H} & 6.8161 & -1.7267 & 18.3705 \\ \mathrm{C} & 7.0874 & -3.7172 & 17.4953 \\ \mathrm{H} & 6.0127 & -4.0167 & 17.5133 \\ \mathrm{H} & 7.5824 & -4.0761 & 18.4287 \\ \mathrm{C} & 8.7394 & -3.5996 & 15.5367 \\ \mathrm{H} & 9.4528 & -4.2417 & 14.9712 \\ \mathrm{H} & 8.1408 & -3.0147 & 14.7994 \\ \mathrm{C} & 9.5383 & -2.6474 & 16.4392 \\ \mathrm{H} & 10.115 & -3.2387 & 17.1893 \\ \mathrm{H} & 10.2472 & -2.0638 & 15.8058 \\ \mathrm{C} & 7.6246 & -5.618 & 16.1563 \\ \mathrm{~N} & 6.8203 & -6.3668 & 16.9177 \\ \mathrm{C} & 6.7365 & -7.684 & 16.7023 \\ \mathrm{~N} & 7.4596 & -8.246 & 15.7285 \\ \mathrm{C} & 8.2458 & -7.508 & 14.9449 \\ \mathrm{~N} & 8.3102 & -6.1968 & 15.1693 \\ \mathrm{C} & 5.3587 & -10.5827 & 18.3587 \\ \mathrm{H} & 5.4183 & -11.694 & 18.2838 \\ \mathrm{H} & 5.9418 & -10.3397 & 19.2777\end{array}$




\begin{tabular}{|c|c|c|c|}
\hline & & & \\
\hline & 7.0637 & & \\
\hline & 0913 & & \\
\hline & & 97 & \\
\hline & & & \\
\hline & 2 & & \\
\hline & & & \\
\hline & & & \\
\hline & & & \\
\hline & 84 & -28. & \\
\hline & & & \\
\hline & & & \\
\hline & -7.1469 & & \\
\hline & & & \\
\hline & & & \\
\hline & & & \\
\hline & -6.8661 & & \\
\hline & -7.8005 & & \\
\hline & & & \\
\hline & -6.2077 & & \\
\hline & -4.5 & & \\
\hline & & & \\
\hline & & & \\
\hline & -4.8408 & & \\
\hline & & & \\
\hline & & & \\
\hline & & & \\
\hline & -6.946 & -23.3 & \\
\hline & 803 & & \\
\hline & & & \\
\hline & -8.0484 & -23 . & \\
\hline & -16 & -26 & \\
\hline & -2.09 & & \\
\hline & -0.6967 & -22. & \\
\hline & & & \\
\hline & & -20 . & 24.607 \\
\hline & & -19.23 & \\
\hline & & -20.54 & \\
\hline
\end{tabular}




$\begin{array}{llll}\mathrm{C} & -7.8065 & -26.084 & 11.0555 \\ \mathrm{H} & -8.525 & -25.9663 & 10.2109 \\ \mathrm{H} & -8.1464 & -25.4136 & 11.8775 \\ \mathrm{C} & -5.8639 & -26.6068 & 9.4973 \\ \mathrm{H} & -14.6635 & -17.8545 & 24.6236 \\ \mathrm{H} & -4.828 & -26.3074 & 9.2177 \\ \mathrm{~N} & -16.0997 & -19.99 & 22.3536 \\ \mathrm{H} & -16.5369 & -25.5425 & 21.5732 \\ \mathrm{H} & -5.158 & -28.1603 & 10.8443 \\ \mathrm{C} & -5.8497 & -24.7113 & 10.9569 \\ \mathrm{~N} & -6.3938 & -23.924 & 11.8873 \\ \mathrm{H} & -7.2511 & -29.5296 & 10.828 \\ \mathrm{C} & -5.759 & -22.8423 & 12.3433 \\ \mathrm{~N} & -10.2903 & -20.4113 & 18.6721 \\ \mathrm{~N} & -4.553 & -22.5537 & 11.8499 \\ \mathrm{C} & -3.9791 & -23.3216 & 10.921 \\ \mathrm{~N} & -2.832 & -23.04 & 10.4659 \\ \mathrm{~N} & -4.6422 & -24.3948 & 10.4845 \\ \mathrm{C} & -15.0739 & -18.8295 & 24.2669 \\ \mathrm{H} & -5.5086 & -28.74 & 9.179 \\ \mathrm{C} & -12.4887 & -21.3211 & 17.9267 \\ \mathrm{H} & -12.5625 & -20.6789 & 17.018 \\ \mathrm{H} & -13.0433 & -22.2637 & 17.7147 \\ \mathrm{C} & -11.0092 & -21.6318 & 18.2193 \\ \mathrm{H} & -10.5419 & -22.1035 & 17.3247 \\ \mathrm{H} & -10.9475 & -22.3623 & 19.0597 \\ \mathrm{C} & -17.5654 & -24.073 & 20.3448 \\ \mathrm{C} & -17.3336 & -25.5233 & 20.7916 \\ \mathrm{C} & -10.8998 & -19.5998 & 19.7594 \\ \mathrm{H} & -10.8428 & -20.2192 & 20.6851 \\ \mathrm{H} & -10.3539 & -18.6484 & 19.9549 \\ \mathrm{H} & -12.3737 & -19.2917 & 19.4454 \\ \mathrm{H} & -18.7768 & 20.3105 \\ \mathrm{H} & -23.4245 & -25.8345 & 18.9334\end{array}$




\begin{tabular}{|c|c|c|c|}
\hline & & & \\
\hline & -15.4373 & & \\
\hline & 14 & & \\
\hline & & & \\
\hline & & & \\
\hline & -17.682 & & \\
\hline & & & \\
\hline & & & \\
\hline & -14.5 & -20 & \\
\hline & -14 . & & \\
\hline & -2.7 & & \\
\hline & & & \\
\hline & 0.2117 & & \\
\hline & -0.91 & & \\
\hline & & & \\
\hline & -15.7 & & \\
\hline & & & \\
\hline & & & \\
\hline & -16.167 & -21 & \\
\hline & -15.9 & & \\
\hline & & & \\
\hline & & & \\
\hline & & & \\
\hline & -17.0625 & & \\
\hline & & & \\
\hline & & & \\
\hline & & & \\
\hline & -2.6178 & & 11. \\
\hline & -1.9977 & & \\
\hline & & -21.39 & \\
\hline & -0.8032 & -22. & \\
\hline & -14 & & \\
\hline & & & 18.0 \\
\hline & -18.0807 & -19.6871 & 22.97 \\
\hline & -7.3 & -20.535 & \\
\hline & & -19.4 & 17.1 \\
\hline & -7.3185 & & 18.1 \\
\hline & -0.5546 & & \\
\hline
\end{tabular}




$\begin{array}{llll}\mathrm{N} & -8.5165 & -19.0181 & 18.62 \\ \mathrm{H} & -6.4968 & -26.5104 & 8.5842 \\ \mathrm{C} & -9.1647 & -20.0998 & 18.1848 \\ \mathrm{~N} & -8.5904 & -20.8469 & 17.2398 \\ \mathrm{H} & -16.7104 & -27.4419 & 19.9535 \\ \mathrm{H} & -17.729 & -26.4485 & 18.8583 \\ \mathrm{~N} & -6.7278 & -17.6554 & 18.5756 \\ \mathrm{~N} & -5.4862 & -15.2222 & 19.4894 \\ \mathrm{C} & -7.3724 & -16.84 & 19.6356 \\ \mathrm{H} & -7.9396 & -17.4822 & 20.35 \\ \mathrm{H} & -8.0813 & -16.1452 & 19.1261 \\ \mathrm{C} & -6.3332 & -16.0172 & 20.4143 \\ \mathrm{H} & -5.6502 & -16.708 & 20.9626 \\ \mathrm{H} & -6.8596 & -15.3745 & 21.1575 \\ \mathrm{C} & -5.1897 & -15.7386 & 18.1295 \\ \mathrm{H} & -5.9035 & -15.238 & 17.4333 \\ \mathrm{H} & -4.147 & -15.4823 & 17.8269 \\ \mathrm{C} & -5.3981 & -17.2622 & 18.0438 \\ \mathrm{H} & -5.2626 & -17.5994 & 16.9895 \\ \mathrm{H} & -4.6363 & -17.7703 & 18.6807 \\ \mathrm{C} & -19.0753 & -10.8048 & 30.0254 \\ \mathrm{C} & -18.6248 & -9.6992 & 30.9928 \\ \mathrm{~N} & -13.3575 & -10.3762 & 26.4115 \\ \mathrm{H} & -16.5226 & -10.2605 & 31.0479 \\ \mathrm{~N} & -17.3865 & -9.9949 & 28.428 \\ \mathrm{H} & -19.5104 & -15.0902 & 24.9919 \\ \mathrm{C} & -17.665 & -15.4183 & 23.8624 \\ \mathrm{~N} & -10.5844 & -10.3233 & 26.8752 \\ \mathrm{C} & -17.1381 & -9.3642 & 30.7951 \\ \mathrm{C} & -12.4592 & -11.0137 & 25.4132 \\ \mathrm{H} & -12.2449 & -10.241 & 24.638 \\ \mathrm{H} & -12.9248 & -11.8872 & 24.9022 \\ \mathrm{C} & -11.1421 & -11.4488 & 26.0812 \\ \mathrm{H} & -10.4363 & -11.8345 & 25.3101 \\ \mathrm{H} & -11.3424 & -12.2792 & 26.7982 \\ \mathrm{C} & -18.2607 & -13.3004 & 25.1033 \\ \mathrm{C} & -18.4276 & -14.8253 & 25.0561 \\ \mathrm{C} & -11.4688 & -9.6919 & 27.8873\end{array}$




\begin{tabular}{|c|c|c|c|}
\hline & & & \\
\hline & & -8.8184 & \\
\hline & & & \\
\hline & & & \\
\hline & & & \\
\hline & & & \\
\hline & & & \\
\hline & & & \\
\hline & & & \\
\hline & -0.9 & & \\
\hline & & & \\
\hline & & & \\
\hline & -17 . & & \\
\hline & & & \\
\hline & & & \\
\hline & -0.7 & & \\
\hline & -19.0 & & \\
\hline & & & \\
\hline & & & \\
\hline & -18.5 & & \\
\hline & -16. & & \\
\hline & & & \\
\hline & -18. & & \\
\hline & -14.566 & & \\
\hline & -7.2 & & \\
\hline & & & \\
\hline & & & \\
\hline & -16 & & \\
\hline & & & \\
\hline & & & \\
\hline & -20 . & & \\
\hline & -8.21 & -5.2 & \\
\hline & -8.7 & & \\
\hline & -8.7793 & & \\
\hline & & -6. & \\
\hline & -9.1 & & \\
\hline & -7.5973 & & 29.7 \\
\hline & -7.3064 & -0.0124 & \\
\hline
\end{tabular}




$$
\begin{aligned}
& \begin{array}{llll}
\text { C } & -7.4953 & -0.1807 & 33.2075
\end{array} \\
& \begin{array}{llll}
\text { C } & -5.8832 & -6.8782 & 27.5675
\end{array} \\
& \begin{array}{llll}
\mathrm{H} & -5.3208 & -6.9999 & 28.5228
\end{array} \\
& \mathrm{H} \quad-5.3219 \quad-7.4265 \quad 26.7763 \\
& \text { C } \quad-5.9761 \quad-5.3826 \quad 27.2123 \\
& \mathrm{H} \quad-4.9534 \quad-4.9444 \quad 27.1581 \\
& \mathrm{H} \quad-19.2386 \quad-8.7829 \quad 30.8224 \\
& \mathrm{H} \quad-16.8318-8.5452 \quad 31.4894 \\
& \text { H } \quad-17.7961 \quad-16.526 \quad 23.8444 \\
& \text { C } \quad \begin{array}{llll}
-9.0642 & -1.8061 & 31.2859
\end{array} \\
& \begin{array}{llll}
\text { C } & -9.2451 & -1.9677 & 32.8016
\end{array} \\
& \mathrm{H} \quad-6.4443 \quad-5.2719 \quad 26.2061 \\
& \begin{array}{llll}
\text { C } & -2.8049 & -2.1522 & 27.9276
\end{array} \\
& \mathrm{H} \quad-2.6694 \quad-1.4659 \quad 27.0592 \\
& \mathrm{H} \quad-3.2003 \quad-3.1154 \quad 27.5319 \\
& \begin{array}{llll}
\text { C } & -3.2839 & -0.2325 & 29.5276
\end{array} \\
& \mathrm{H} \quad-4.0176 \quad 0.1622 \quad 30.2671 \\
& \begin{array}{llll}
\mathrm{H} & -1.5384 & 0.4797 & 30.6342
\end{array} \\
& \begin{array}{llll}
\text { C } & -4.8817 & -1.9611 & 29.0996
\end{array} \\
& \begin{array}{llll}
\mathrm{N} & -5.284 & -3.0861 & 28.5037
\end{array} \\
& \begin{array}{llll}
\text { C } & -6.4913 & -3.6037 & 28.7388
\end{array} \\
& \mathrm{H} \quad-18.7697 \quad-12.903 \quad 26.0111 \\
& \mathrm{H} \quad-18.7497-12.840524 .2128 \\
& \text { H } \quad-18.0911-15.025322 .9089 \\
& \text { H } \quad-18.0337-15.269326 .0016 \\
& \begin{array}{llll}
\mathrm{N} & -7.3027 & -2.9704 & 29.5881
\end{array} \\
& \begin{array}{llll}
\text { C } & -6.9318 & -1.8448 & 30.2015
\end{array} \\
& \begin{array}{llll}
\mathrm{N} & -7.7151 & -1.2599 & 31.0057
\end{array} \\
& \begin{array}{llll}
\mathrm{N} & -5.7175 & -1.3536 & 29.945
\end{array} \\
& \mathrm{H} \quad-2.0818 \quad-1.177 \quad 31.0731 \\
& \mathrm{H} \quad 0.0445 \quad-1.2834 \quad 29.7598 \\
& \mathrm{H} \quad-1.5938 \quad-3.147 \quad 29.4343 \\
& \mathrm{H} \quad-6.2552 \quad 0.2843 \quad 31.4747 \\
& \mathrm{H} \quad-7.9687 \quad 0.8002 \quad 31.3131 \\
& \begin{array}{llll}
\mathrm{H} & -0.7065 & -0.3549 & 28.419
\end{array} \\
& \mathrm{H} \quad-7.2821 \quad 0.7831 \quad 33.7293 \\
& \text { H } \quad-9.2592 \quad-2.7779 \quad 30.7777 \\
& \text { H } \quad-9.8014 \quad-1.0695 \quad 30.889
\end{aligned}
$$




\begin{tabular}{|c|c|c|c|}
\hline & & & \\
\hline & -16.3098 & & \\
\hline & & & \\
\hline & 411 & & \\
\hline & -8.5638 & & \\
\hline & -10.29 & & \\
\hline & -3.1 & & \\
\hline & -9.4 & & \\
\hline & & & \\
\hline & -7.3 & & \\
\hline & -3.0 & & \\
\hline & & & \\
\hline & -19 . & -9.5 & \\
\hline & -7.6 & -8.4 & \\
\hline & & & \\
\hline & -9.0 & & \\
\hline & -9.65 & & \\
\hline & & & \\
\hline & & & \\
\hline & -5.2523 & & \\
\hline & -4.60 & & \\
\hline & & & \\
\hline & -4.8 & & \\
\hline & -3.7505 & -10.4 & \\
\hline & & -9.9 & \\
\hline & & & \\
\hline & & & \\
\hline & -5.4 & & \\
\hline & & & \\
\hline & -7.86 & & \\
\hline & & & \\
\hline & -4.8 & & \\
\hline & & & \\
\hline & -3.80 & & \\
\hline & & & \\
\hline & -5.0 & & \\
\hline & & & \\
\hline & -1.4969 & & \\
\hline
\end{tabular}




$\begin{array}{llll}\mathrm{H} & -1.1106 & -12.126 & 15.8969 \\ \mathrm{H} & -1.7079 & -10.4703 & 16.2394 \\ \mathrm{C} & -2.7687 & -12.0983 & 17.2845 \\ \mathrm{H} & -3.6421 & -11.8973 & 16.6199 \\ \mathrm{C} & -2.4572 & -10.2309 & 18.986 \\ \mathrm{H} & -2.9122 & -9.4809 & 18.297 \\ \mathrm{H} & -2.7039 & -9.9131 & 20.0255 \\ \mathrm{C} & -0.9306 & -10.2885 & 18.779 \\ \mathrm{H} & -0.5535 & -9.2788 & 18.4887 \\ \mathrm{H} & -0.4464 & -10.5819 & 19.7402 \\ \mathrm{H} & -2.6066 & -13.1993 & 17.365 \\ \mathrm{C} & 3.0396 & -10.8627 & 17.8735 \\ \mathrm{~N} & 3.4036 & -11.7324 & 16.9245 \\ \mathrm{C} & 2.4655 & -12.3934 & 16.2493 \\ \mathrm{Cl} & 2.9408 & -13.4971 & 15.0229 \\ \mathrm{~N} & 1.1701 & -12.2267 & 16.5099 \\ \mathrm{C} & 0.7921 & -11.3714 & 17.4661 \\ \mathrm{~N} & 1.7361 & -10.6939 & 18.1357\end{array}$




$$
\begin{aligned}
& \text { Parameters of } \mathbf{G}_{\mathbf{4}}-\mathbf{N H} \text { ( } \mathrm{R}=\text { piperidine) } \\
& \text { C } \quad 13.1727-19.9527 \quad 4.5363 \\
& \begin{array}{llll}
\text { C } & 14.5568 & -19.3474 & 4.2549
\end{array} \\
& \begin{array}{llll}
\mathrm{N} & 12.1784 & -15.905 & 9.8802
\end{array} \\
& \text { H } \quad 14.0394 \quad-17.2975 \quad 4.7731 \\
& \mathrm{~N} \quad 13.3095 \quad-19.02526 .8133 \\
& \begin{array}{llll}
\text { H } & 7.293 & -21.2218 & 6.9436
\end{array} \\
& \begin{array}{llll}
\text { C } & 6.5407 & -19.815 & 8.4433
\end{array} \\
& \text { N } \quad 12.1844 \quad-13.1124 \quad 10.1809 \\
& \text { C } \quad 14.7806 \quad-18.07255 .0831 \\
& \begin{array}{llll}
\text { C } & 11.2187 & -15.312 & 10.846
\end{array} \\
& \text { H } \quad 11.7124 \quad-15.341911 .8455 \\
& \text { H } \quad 10.2675 \quad-15.886 \quad 10.9276 \\
& \text { C } \quad 10.9243 \quad-13.8511 \quad 10.4604 \\
& \text { H } \quad 10.3061 \quad-13.3718 \quad 11.2539 \\
& \text { H } \quad 10.3347 \quad-13.8343 \quad 9.5138 \\
& \text { C } \quad 8.9612 \quad-20.48648 .1497 \\
& \begin{array}{llll}
\text { C } & 7.6136 & -20.277 & 7.4449
\end{array} \\
& \text { C } \quad 13.1723 \quad-13.7317 \quad 9.2569 \\
& \begin{array}{llll}
\mathrm{H} & 12.708 & -13.7189 & 8.243
\end{array} \\
& \text { H } \quad 14.1307 \quad-13.167 \quad 9.1974 \\
& \begin{array}{llll}
\text { C } & 13.46 & -15.1871 & 9.6644
\end{array} \\
& \text { H } \quad 14.116 \quad-15.67488 .9077 \\
& \begin{array}{llll}
\text { C } & 22.5881 & -8.8659 & 6.0873
\end{array} \\
& \begin{array}{llll}
\text { C } & 8.3211 & -18.7335 & 9.8785
\end{array} \\
& \text { C } 6.9753 \quad-18.53149 .168 \\
& \text { H } \quad 14.0055 \quad-15.203510 .6369 \\
& \begin{array}{llll}
\text { C } & 20.5069 & -2.581 & 10.0549
\end{array} \\
& \text { C } \quad 19.5247 \quad-1.8768 \quad 11.0042 \\
& \begin{array}{llll}
\mathrm{N} & 17.4399 & -8.5508 & 10.8614
\end{array} \\
& \begin{array}{llll}
\text { C } & 14.6127 & -18.3584 & 6.5821
\end{array} \\
& \text { H } \quad \begin{array}{llll}
\text { H } & 15.4041 & -19.0638 & 6.9277
\end{array} \\
& \text { H } \quad 14.7321-17.41467 .1615 \\
& \text { C } \quad 12.9977-20.24596 .033 \\
& \begin{array}{llll}
\mathrm{H} & 17.6525 & -2.274 & 12.0678
\end{array} \\
& \text { H } \quad 11.9727 \quad-20.6383 \quad 6.2228 \\
& \text { N } \quad 19.8304 \quad-4.7239 \quad 11.0533 \\
& \begin{array}{llll}
\mathrm{H} & 21.8001 & -6.8401 & 6.1983
\end{array}
\end{aligned}
$$




$\begin{array}{llll}\text { H } & 12.3838 & -19.2337 & 4.2095 \\ \mathrm{C} & 12.4827 & -18.5622 & 7.6525 \\ \mathrm{~N} & 12.7632 & -17.4602 & 8.3512 \\ \mathrm{H} & 14.6544 & -19.1145 & 3.1672 \\ \mathrm{C} & 11.8996 & -16.9573 & 9.2357 \\ \mathrm{~N} & 14.7927 & -9.4403 & 10.5789 \\ \mathrm{~N} & 10.7334 & -17.5804 & 9.4174 \\ \mathrm{C} & 10.4222 & -18.6846 & 8.7349 \\ \mathrm{~N} & 9.3109 & -19.2644 & 8.9119 \\ \mathrm{~N} & 11.3081 & -19.1619 & 7.858 \\ \mathrm{C} & 18.339 & -2.7895 & 11.3542 \\ \mathrm{H} & 13.0338 & -20.8889 & 3.9439 \\ \mathrm{C} & 17.2193 & -9.9291 & 10.3521 \\ \mathrm{H} & 17.2649 & -10.6061 & 11.2373 \\ \mathrm{H} & 18.005 & -10.2615 & 9.6358 \\ \mathrm{C} & 15.8302 & -10.0355 & 9.6965 \\ \mathrm{H} & 15.6184 & -11.0965 & 9.4312 \\ \mathrm{H} & 15.8206 & -9.4421 & 8.7523 \\ \mathrm{C} & 22.5194 & -7.4248 & 8.1649 \\ \mathrm{C} & 22.6407 & -7.4311 & 6.6346 \\ \mathrm{C} & 15.0255 & -8.0687 & 11.1038 \\ \mathrm{H} & 14.9904 & -7.3827 & 10.2254 \\ \mathrm{H} & 14.2432 & -7.734 & 11.8228 \\ \mathrm{C} & 16.4103 & -7.9792 & 11.7669 \\ \mathrm{H} & 16.6216 & -6.9273 & 12.0661 \\ \mathrm{H} & 15.3475 & -20.0953 & 4.5019 \\ \mathrm{H} & 15.7976 & -17.6569 & 4.8841 \\ \mathrm{H} & 5.5761 & -19.6401 & 7.9086 \\ \mathrm{C} & 21.1713 & -9.581 & 8.0564 \\ \mathrm{C} & 21.2982 & -9.576 & 6.5266 \\ \mathrm{H} & 16.4211 & -8.5981 & 12.6945 \\ \mathrm{C} & 18.8232 & -4.1167 & 11.9542 \\ \mathrm{H} & 19.3319 & -3.9376 & 12.9293 \\ \mathrm{H} & 17.9521 & -4.7813 & 12.154 \\ \mathrm{C} & 20.9995 & -3.9038 & 10.6585 \\ \mathrm{H} & 21.675 & -4.4208 & 9.9395 \\ \mathrm{H} & 21.3751 & -1.9142 & 9.8349 \\ \mathrm{C} & 19.6894 & -5.9057 & 10.6229\end{array}$




$$
\begin{aligned}
& \begin{array}{llll}
\mathrm{N} & 18.6283 & -6.635 & 10.9735
\end{array} \\
& \begin{array}{llll}
\text { C } & 18.4574 & -7.8794 & 10.5233
\end{array} \\
& \text { H } \quad 9.7361 \quad-20.77657 .4039 \\
& \text { H } \quad 8.8821 \quad-21.31848 .8879 \\
& \text { H } \quad 6.3582 \quad-20.62179 .1926 \\
& \text { H } \quad 7.7333 \quad-19.50366 .6489 \\
& \begin{array}{llll}
\mathrm{N} & 19.3784 & -8.396 & 9.7075
\end{array} \\
& \begin{array}{llll}
\text { C } & 20.4536 & -7.6949 & 9.3406
\end{array} \\
& \begin{array}{llll}
\mathrm{N} & 21.3219 & -8.1988 & 8.5695
\end{array} \\
& \begin{array}{llll}
\mathrm{N} & 20.5933 & -6.4517 & 9.8066
\end{array} \\
& \begin{array}{llll}
\mathrm{H} & 19.9952 & -2.79 & 9.0851
\end{array} \\
& \text { H } \quad 19.1515 \quad-0.9353 \quad 10.5341 \\
& \begin{array}{llll}
\mathrm{H} & 17.754 & -3.0014 & 10.4275
\end{array} \\
& \text { H } \quad 22.5096 \quad-6.3749 \quad 8.5368 \\
& \begin{array}{llll}
\mathrm{H} & 23.4019 & -7.9319 & 8.6203
\end{array} \\
& \text { H } \quad 20.0586 \quad-1.5831 \quad 11.9383 \\
& \begin{array}{llll}
\mathrm{H} & 23.5914 & -6.9345 & 6.324
\end{array} \\
& \text { H } \quad 20.2084 \quad-10.0566 \quad 8.3519 \\
& \text { H } \quad 21.9937-10.18498 .5062 \\
& \text { H } \quad 8.6424 \quad-17.780410 .3574 \\
& \text { H } \quad 8.2167 \quad-19.493910 .6874 \\
& \text { H } 6.1957-18.22239 .9052 \\
& \text { H } \quad 7.0747 \quad-17.7048 \quad 8.4244 \\
& \text { H } \quad 20.4189 \quad-9.0462 \quad 6.0881 \\
& \begin{array}{llll}
\mathrm{H} & 21.2825 & -10.6227 & 6.1378
\end{array} \\
& \mathrm{H} \quad 21.5871 \quad-3.7069 \quad 11.5856 \\
& \text { C } \quad 12.3863 \quad-11.962810 .6702 \\
& \begin{array}{llll}
\mathrm{N} & 11.483 & -11.3904 & 11.471
\end{array} \\
& \text { C } \quad 11.6878 \quad-10.1677 \quad 11.9656 \\
& \begin{array}{llll}
\mathrm{N} & 4.5283 & -10.35 & 19.5431
\end{array} \\
& \text { N } \quad 12.8122 \quad-9.5216 \quad 11.6525 \\
& \text { H } \quad 13.7204 \quad-21.03126 .3562 \\
& \text { C } \quad 13.7339 \quad-10.0724 \quad 10.8618 \\
& \begin{array}{llll}
\mathrm{N} & 13.5049 & -11.2944 & 10.3774
\end{array} \\
& \begin{array}{llll}
\mathrm{H} & 22.65 & -8.8485 & 4.9726
\end{array} \\
& \begin{array}{llll}
\mathrm{H} & 23.4725 & -9.438 & 6.4559
\end{array} \\
& \begin{array}{llll}
\mathrm{N} & 10.8395 & -9.6081 & 12.7188
\end{array} \\
& \begin{array}{llll}
\mathrm{N} & 9.0062 & -8.3452 & 14.5401
\end{array}
\end{aligned}
$$




$\begin{array}{llll}\mathrm{C} & 11.0868 & -8.2231 & 13.1944 \\ \mathrm{H} & 11.6129 & -7.6173 & 12.4194 \\ \mathrm{H} & 11.7322 & -8.297 & 14.1015 \\ \mathrm{C} & 9.7675 & -7.5257 & 13.5641 \\ \mathrm{H} & 9.1298 & -7.4252 & 12.6545 \\ \mathrm{H} & 9.9911 & -6.5068 & 13.9569 \\ \mathrm{C} & 9.1058 & -9.8264 & 14.4982 \\ \mathrm{H} & 9.8476 & -10.1237 & 15.2767 \\ \mathrm{H} & 8.1211 & -10.2943 & 14.7356 \\ \mathrm{C} & 9.5994 & -10.318 & 13.1233 \\ \mathrm{H} & 9.7537 & -11.4221 & 13.1514 \\ \mathrm{H} & 8.8281 & -10.0846 & 12.3521 \\ \mathrm{C} & 19.3806 & 5.4366 & 18.1095 \\ \mathrm{C} & 18.5472 & 6.5645 & 18.7377 \\ \mathrm{~N} & 14.1189 & 1.1574 & 18.1173 \\ \mathrm{H} & 16.76 .1285 & 17.6722 \\ \mathrm{~N} & 17.6762 & 3.8403 & 18.8902 \\ \mathrm{H} & 21.4431 & -0.361 & 15.6703 \\ \mathrm{C} & 19.8726 & -1.8209 & 15.2335 \\ \mathrm{~N} & 11.4019 & 1.1068 & 17.4084 \\ \mathrm{C} & 17.0506 & 6.216 & 18.7284 \\ \mathrm{C} & 13.5389 & -0.1147 & 17.6101 \\ \mathrm{H} & 13.2112 & -0.6945 & 18.5049 \\ \mathrm{H} & 14.2745 & -0.7377 & 17.0519 \\ \mathrm{C} & 12.3217 & 0.1707 & 16.712 \\ \mathrm{H} & 11.8347 & -0.7859 & 16.4136 \\ \mathrm{H} & 12.6545 & 0.6805 & 15.7776 \\ \mathrm{C} & 19.747 & -0.0273 & 17.0081 \\ \mathrm{C} & 20.3281 & -0.4119 & 15.6405 \\ \mathrm{C} & 11.9659 & 2.3981 & 17.8742 \\ \mathrm{H} & 12.2677 & 2.9609 & 16.96 \\ \mathrm{H} & 11.2255 & 3.0252 & 18.4215 \\ \mathrm{C} & 13.2033 & 2.1426 & 18.7527 \\ \mathrm{H} & 13.7009 & 3.111 & 18.9884 \\ \mathrm{C} & 6.2025 & 10.0592 & 23.1007 \\ \mathrm{C} & 17.7369 & -1.5324 & 16.552 \\ \mathrm{C} & 18.3394 & -1.9007 & 15.189 \\ \mathrm{H} & 12.8766 & 1.6871 & 19.7169\end{array}$




\begin{tabular}{|c|c|c|c|}
\hline & & & \\
\hline & -0.7273 & & \\
\hline & & & \\
\hline & & & \\
\hline & & & \\
\hline & & & \\
\hline & & & \\
\hline & & & \\
\hline & & & \\
\hline & & & \\
\hline & & & \\
\hline & & & \\
\hline & 17.1 & & \\
\hline & & & \\
\hline & & & \\
\hline & & & \\
\hline & 7.3653 & 2.055 & \\
\hline & & & \\
\hline & & 0.6 & \\
\hline & 18.2 & -0.2338 & \\
\hline & 17.5 & 1.80 & \\
\hline & & & \\
\hline & 20.4664 & 5.69 & \\
\hline & & 3.5632 & \\
\hline & & & \\
\hline & & & \\
\hline & & & \\
\hline & 8.95 & & \\
\hline & 7.3 & & \\
\hline & 4.6449 & & \\
\hline & 4.738 & & \\
\hline & 5.950 & 1.7 & \\
\hline & 5.322 & 2.3987 & \\
\hline & 5.6882 & 0.6642 & \\
\hline & & & \\
\hline & & & \\
\hline & 18.8832 & & \\
\hline & 16.461 & 7.0348 & \\
\hline
\end{tabular}




$\begin{array}{llll}\text { H } & 20.2999 & -2.0815 & 14.2365 \\ \mathrm{C} & 6.9387 & 7.6397 & 23.1247 \\ \mathrm{C} & 7.0219 & 8.9786 & 22.3783 \\ \mathrm{H} & 6.1872 & 1.151 & 21.6019 \\ \mathrm{C} & 1.5079 & 3.0749 & 22.613 \\ \mathrm{H} & 1.2114 & 2.5356 & 23.5429 \\ \mathrm{H} & 2.2454 & 2.4342 & 22.0777 \\ \mathrm{C} & 1.156 & 5.3572 & 23.6802 \\ \mathrm{H} & 1.6441 & 6.3335 & 23.902 \\ \mathrm{H} & -0.8086 & 6.2397 & 23.306 \\ \mathrm{C} & 3.2831 & 4.6641 & 22.8315 \\ \mathrm{~N} & 4.0981 & 3.7877 & 22.2399 \\ \mathrm{C} & 5.382 & 4.0748 & 22.0163 \\ \mathrm{H} & 20.0419 & 1.0184 & 17.2538 \\ \mathrm{H} & 20.17 & -0.6918 & 17.7976 \\ \mathrm{H} & 20.2664 & -2.5696 & 15.9612 \\ \mathrm{H} & 19.9836 & 0.3224 & 14.8735 \\ \mathrm{~N} & 5.8473 & 5.2651 & 22.4008 \\ \mathrm{C} & 5.0601 & 6.1631 & 22.9966 \\ \mathrm{~N} & 5.5171 & 7.2883 & 23.3541 \\ \mathrm{~N} & 3.7805 & 5.8454 & 23.2044 \\ \mathrm{H} & 0.2446 & 6.1089 & 21.8547 \\ \mathrm{H} & -1.5996 & 4.4231 & 21.7392 \\ \mathrm{H} & 0.6058 & 3.7651 & 20.7594 \\ \mathrm{H} & 3.5806 & 7.9658 & 24.0805 \\ \mathrm{H} & 5.0218 & 8.4082 & 25.0576 \\ \mathrm{H} & -1.1208 & 3.7514 & 23.3342 \\ \mathrm{H} & 4.1604 & 10.4093 & 23.8077 \\ \mathrm{H} & 7.4981 & 6.8591 & 22.5604 \\ \mathrm{H} & 7.4158 & 7.7319 & 24.1283 \\ \mathrm{H} & 16.6254 & -1.5394 & 16.4783 \\ \mathrm{H} & 18.0295 & -2.2942 & 17.3121 \\ \mathrm{H} & 18.0133 & -2.9265 & 14.8927 \\ \mathrm{H} & 17.9565 & -1.1946 & 14.4141 \\ \mathrm{H} & 6.625 & 8.8463 & 21.3433 \\ \mathrm{H} & 8.0872 & 9.3 & 22.286 \\ \mathrm{H} & 0.8444 & 4.912 & 24.6538 \\ \mathrm{C} & 10.1977 & 0.7936 & 17.636\end{array}$




$\begin{array}{llll}\mathrm{N} & 9.7168 & -0.3743 & 17.2107 \\ \mathrm{C} & 8.4568 & -0.7397 & 17.4542 \\ \mathrm{~N} & 6.2975 & -8.6176 & 18.1218 \\ \mathrm{~N} & 7.6626 & 0.0818 & 18.1441 \\ \mathrm{H} & 19.4924 & 4.1709 & 19.8873 \\ \mathrm{C} & 8.1167 & 1.2635 & 18.5721 \\ \mathrm{~N} & 9.3812 & 1.6056 & 18.31 \\ \mathrm{H} & 6.2512 & 11.0171 & 22.5289 \\ \mathrm{H} & 6.6474 & 10.2545 & 24.1055 \\ \mathrm{~N} & 8.0527 & -1.8559 & 17.0175 \\ \mathrm{~N} & 7.4258 & -4.5717 & 16.4131 \\ \mathrm{C} & 6.6818 & -2.349 & 17.3081 \\ \mathrm{H} & 6.0529 & -2.0798 & 16.4272 \\ \mathrm{H} & 6.2471 & -1.8567 & 18.2091 \\ \mathrm{C} & 6.6764 & -3.8806 & 17.4928 \\ \mathrm{H} & 5.6285 & -4.2625 & 17.5262 \\ \mathrm{H} & 7.1878 & -4.1226 & 18.4547 \\ \mathrm{C} & 8.2078 & -3.7719 & 15.4366 \\ \mathrm{H} & 8.9245 & -4.3814 & 14.8401 \\ \mathrm{H} & 7.4784 & -3.3167 & 14.7264 \\ \mathrm{C} & 8.9746 & -2.6713 & 16.1861 \\ \mathrm{H} & 9.7187 & -3.1427 & 16.8714 \\ \mathrm{H} & 9.5047 & -2.0273 & 15.4447 \\ \mathrm{C} & 7.4618 & -5.8359 & 16.3858 \\ \mathrm{~N} & 6.8044 & -6.5657 & 17.2914 \\ \mathrm{C} & 6.8968 & -7.8988 & 17.271 \\ \mathrm{~N} & 4.7418 & -16.6615 & 16.303 \\ \mathrm{~N} & 7.6454 & -8.4986 & 16.3409 \\ \mathrm{C} & 8.2946 & -7.783 & 15.4216 \\ \mathrm{~N} & 8.1907 & -6.4548 & 15.4555 \\ \mathrm{C} & 5.9333 & -10.6723 & 19.3868 \\ \mathrm{H} & 6.1148 & -11.7701 & 19.4615 \\ \mathrm{H} & 6.50948 & -10.2469 & 20.2613 \\ \mathrm{H} & -10.0816 & 18.0959 \\ \mathrm{H} & -10.3127 & 18.0316 \\ \mathrm{H} & -8.0287 & 19.1362 \\ \mathrm{H} & -7.9499 & 20.095 \\ \mathrm{H} & & \\ \mathrm{H} & & & \end{array}$




$$
\begin{array}{llll}
\mathrm{C} & 4.178 & -8.9596 & 19.2931 \\
\mathrm{H} & 3.5118 & -8.6082 & 20.1156 \\
\mathrm{H} & 3.6058 & -8.8797 & 18.3373 \\
\mathrm{H} & 5.9819 & -10.499 & 17.206 \\
\mathrm{C} & -5.636 & -27.7187 & 9.6813 \\
\mathrm{C} & -7.0889 & -28.0623 & 10.0449 \\
\mathrm{~N} & -5.6803 & -22.1554 & 13.5846 \\
\mathrm{H} & -6.9635 & -27.4676 & 12.1351 \\
\mathrm{~N} & -6.052 & -25.4281 & 10.4884 \\
\mathrm{H} & 0.2409 & -24.914 & 9.318 \\
\mathrm{C} & 0.6316 & -23.3006 & 10.7452 \\
\mathrm{~N} & -6.323 & -21.4585 & 16.227 \\
\mathrm{C} & -7.5734 & -27.2183 & 11.2339 \\
\mathrm{C} & -4.9134 & -21.0578 & 14.2307 \\
\mathrm{H} & -5.4256 & -20.1071 & 13.9523 \\
\mathrm{H} & -3.8606 & -20.9898 & 13.8731 \\
\mathrm{C} & -4.9328 & -21.2178 & 15.7608 \\
\mathrm{H} & -4.453 & -20.3358 & 16.2434 \\
\mathrm{H} & -4.3399 & -22.1175 & 16.0483 \\
\mathrm{C} & -1.6151 & -23.8267 & 9.7085 \\
\mathrm{C} & -0.2698 & -24.4044 & 10.1703 \\
\mathrm{C} & -7.0715 & -22.5786 & 15.6011 \\
\mathrm{H} & -6.5394 & -23.5169 & 15.8841 \\
\mathrm{H} & -8.1195 & -22.6651 & 15.9688 \\
\mathrm{C} & -7.0606 & -22.4208 & 14.0697 \\
\mathrm{H} & -7.5253 & -23.3161 & 13.5973 \\
\mathrm{C} & -16.7007 & -26.0177 & 19.5705 \\
\mathrm{C} & -1.4087 & -21.9804 & 11.4466 \\
\mathrm{C} & -0.0642 & -22.5653 & 11.9013 \\
\mathrm{H} & -7.673 & -21.533 & 13.7857 \\
\mathrm{C} & -16.6264 & -20.1274 & 24.5377 \\
\mathrm{C} & -15.9805 & -18.8269 & 25.041 \\
\mathrm{~N} & -12.6695 & -20.3094 & 19.0613 \\
\mathrm{C} & -7.4358 & -25.7197 & 10.9315 \\
\mathrm{H} & -8.1113 & -25.4363 & 10.0908 \\
\mathrm{H} & -7.7456 & -25.1268 & 11.8222 \\
\mathrm{C} & -5.4891 & -26.2225 & 9.3709 \\
\mathrm{H} & -14.2908 & -17.5126 & 24.5819
\end{array}
$$




\begin{tabular}{|c|c|c|c|}
\hline & & & \\
\hline & & & \\
\hline & & & \\
\hline & 705 & -27 . & \\
\hline & & -24 & \\
\hline & -5.8 & -23. & \\
\hline & $-7.1^{\prime}$ & -29 . & \\
\hline & 33 & & \\
\hline & & & \\
\hline & -3.9 & -22 & \\
\hline & & & \\
\hline & & & \\
\hline & -4.12 & & \\
\hline & & & \\
\hline & -5.3 & & \\
\hline & & & \\
\hline & & & \\
\hline & & & \\
\hline & & & \\
\hline & & & \\
\hline & & & \\
\hline & & & \\
\hline & & & \\
\hline & & & \\
\hline & & & \\
\hline & & & \\
\hline & & & \\
\hline & -12.3 & & 20 \\
\hline & -7.7 & & \\
\hline & & & \\
\hline & 1.59 & & \\
\hline & & & \\
\hline & & & \\
\hline & & & \\
\hline & & & \\
\hline & -15 . & -17 & 22.532 \\
\hline & & & 22.152 \\
\hline & -16.9591 & -20.03 & 23.0 \\
\hline
\end{tabular}




\begin{tabular}{|c|c|c|c|}
\hline & & & \\
\hline & -17.5516 & & \\
\hline & & & \\
\hline & & -19.9445 & \\
\hline & & -20 & \\
\hline & -2.2715 & -24.6477 & \\
\hline & & & \\
\hline & & & \\
\hline & & & \\
\hline & -14 & -21. & \\
\hline & & & \\
\hline & & & \\
\hline & -15 & & \\
\hline & & & \\
\hline & & & \\
\hline & & & \\
\hline & & & \\
\hline & & & 19.5 \\
\hline & & & \\
\hline & & & \\
\hline & & & \\
\hline & & & \\
\hline & -1.9 & -21 . & \\
\hline & -1.242 & -21 . & \\
\hline & 0.5 & & \\
\hline & & & \\
\hline & & & \\
\hline & & & 18.0 \\
\hline & & & \\
\hline & & & \\
\hline & & & \\
\hline & -6.74 & -18 & \\
\hline & 0.123 & & 18.4 \\
\hline & -7.9968 & -19.1 & \\
\hline & & -25. & \\
\hline & -8.6948 & -20 . & \\
\hline & -8.1133 & -20.93 & 17.50 \\
\hline & -16.5465 & -27.06 & \\
\hline
\end{tabular}




$\begin{array}{llll}\mathrm{H} & -17.513 & -26.0529 & 18.806 \\ \mathrm{~N} & -6.114 & -17.9209 & 19.0382 \\ \mathrm{~N} & -4.8803 & -15.4843 & 19.9191 \\ \mathrm{C} & -6.7924 & -17.0647 & 20.0445 \\ \mathrm{H} & -7.3906 & -17.6848 & 20.7534 \\ \mathrm{H} & -7.4747 & -16.3811 & 19.4857 \\ \mathrm{C} & -5.7814 & -16.2223 & 20.839 \\ \mathrm{H} & -5.135 & -16.8959 & 21.4495 \\ \mathrm{H} & -6.3301 & -15.5425 & 21.5311 \\ \mathrm{C} & -4.4532 & -16.1055 & 18.6398 \\ \mathrm{H} & -5.0542 & -15.6264 & 17.8308 \\ \mathrm{H} & -3.3696 & -15.9138 & 18.4549 \\ \mathrm{C} & -4.7198 & -17.6216 & 18.6246 \\ \mathrm{H} & -4.488 & -18.0326 & 17.6145 \\ \mathrm{H} & -4.0537 & -18.1191 & 19.3679 \\ \mathrm{C} & -18.8566 & -10.2354 & 29.709 \\ \mathrm{C} & -18.4206 & -9.0929 & 30.6399 \\ \mathrm{~N} & -13.1227 & -9.8904 & 26.1284 \\ \mathrm{H} & -16.3101 & -9.6211 & 30.713 \\ \mathrm{~N} & -17.1752 & -9.4603 & 28.0885 \\ \mathrm{H} & -19.212 & -14.7155 & 24.8125 \\ \mathrm{C} & -17.3536 & -15.0526 & 23.7074 \\ \mathrm{~N} & -10.3669 & -9.8751 & 26.6739 \\ \mathrm{C} & -16.9389 & -8.7431 & 30.4305 \\ \mathrm{C} & -12.2093 & -10.573 & 25.1751 \\ \mathrm{H} & -11.9523 & -9.8276 & 24.3865 \\ \mathrm{H} & -12.6765 & -11.4499 & 24.6716 \\ \mathrm{C} & -10.9283 & -11.0162 & 25.9039 \\ \mathrm{H} & -10.2098 & -11.4487 & 25.1705 \\ \mathrm{H} & -11.1738 & -11.8139 & 26.6436 \\ \mathrm{C} & -17.9836 & -12.9089 & 24.8867 \\ \mathrm{C} & -18.1328 & -14.4366 & 24.8785 \\ \mathrm{H} & -11.266 & -9.176 & 27.6273 \\ \mathrm{H} & -10.7898 & -8.2878 & 28.1023 \\ \mathrm{H} & -0.3284 & 33.4438\end{array}$




$$
\begin{aligned}
& \text { C } \quad-15.6887-13.155423 .7922 \\
& \text { C } \quad-15.8665-14.679923 .7977 \\
& \mathrm{H} \quad-12.3609-7.9425 \quad 26.2072 \\
& \begin{array}{llll}
\text { C } & -1.1933 & -0.6836 & 30.3835
\end{array} \\
& \begin{array}{llll}
\text { C } & -0.1994 & -1.3486 & 29.4185
\end{array} \\
& \begin{array}{llll}
\mathrm{N} & -6.3854 & -4.3549 & 28.0163
\end{array} \\
& \text { C } \quad-16.6653 \quad-8.3791 \quad 28.9644 \\
& \mathrm{H} \quad-17.2186-7.4505 \quad 28.6905 \\
& \text { H } \quad-15.5798 \quad-8.1732 \quad 28.822 \\
& \text { C } \quad-18.5894 \quad-9.8763 \quad 28.2405 \\
& \mathrm{H} \quad-0.0984 \quad-3.0395 \quad 28.0313 \\
& \mathrm{H} \quad-18.8687-10.732627 .5851 \\
& \begin{array}{llll}
\mathrm{N} & -3.0196 & -1.4793 & 28.935
\end{array} \\
& \mathrm{H} \quad-6.1572 \quad-0.8002 \quad 33.5625 \\
& \text { H } \quad-18.2869-11.159429 .9691 \\
& \text { C } \quad-16.4192-10.023627 .2439 \\
& \text { N } \quad-15.1435 \quad-9.6519 \quad 27.1152 \\
& \text { H } \quad-18.5943 \quad-9.3842 \quad 31.7038 \\
& \text { C } \quad-14.3312-10.248526 .2406 \\
& \begin{array}{llll}
\mathrm{N} & -6.9402 & -7.0784 & 27.6227
\end{array} \\
& \mathrm{~N} \quad-14.8216-11.229325 .4798 \\
& \text { C } \quad-16.0957-11.616125 .5727 \\
& \mathrm{~N} \quad-16.5525 \quad-12.526324 .8203 \\
& \mathrm{~N} \quad-16.8795-11.003526 .4627 \\
& \begin{array}{llll}
\text { C } & -0.8244 & -2.5799 & 28.7443
\end{array} \\
& \mathrm{H} \quad-19.9401-10.461229 .8568 \\
& \begin{array}{llll}
\text { C } & -7.7836 & -4.8218 & 28.2046
\end{array} \\
& \begin{array}{llll}
\mathrm{H} & -8.3033 & -4.6737 & 27.229
\end{array} \\
& \mathrm{H} \quad-8.3415 \quad-4.24 \quad 28.9733 \\
& \begin{array}{llll}
\text { C } & -7.7955 & -6.3161 & 28.57
\end{array} \\
& \mathrm{H} \quad-8.8451 \quad-6.6864 \quad 28.6123 \\
& \mathrm{H} \quad-7.3514 \quad-6.4552 \quad 29.5835 \\
& \begin{array}{llll}
\text { C } & -6.5485 & 0.2144 & 31.68
\end{array} \\
& \begin{array}{llll}
\text { C } & -6.8062 & 0.0242 & 33.1814
\end{array} \\
& \begin{array}{llll}
\text { C } & -5.5406 & -6.6166 & 27.4327
\end{array} \\
& \text { H } \quad-5.0193 \quad-6.7739 \quad 28.406 \\
& \mathrm{H} \quad-4.9891 \quad-7.1985 \quad 26.6589 \\
& \begin{array}{llll}
\text { C } & -5.521 & -5.1185 & 27.0785
\end{array}
\end{aligned}
$$




$\begin{array}{llll}\mathrm{H} & -4.4703 & -4.7493 & 27.0533 \\ \mathrm{H} & -19.0473 & -8.1912 & 30.4406 \\ \mathrm{H} & -16.6446 & -7.8967 & 31.0966 \\ \mathrm{H} & -17.4732 & -16.1618 & 23.7157 \\ \mathrm{C} & -8.4422 & -1.4036 & 31.1602 \\ \mathrm{C} & -8.6918 & -1.5863 & 32.6639 \\ \mathrm{H} & -5.9524 & -4.9734 & 26.0604 \\ \mathrm{C} & -2.1173 & -2.2021 & 28.0078 \\ \mathrm{H} & -1.8893 & -1.5053 & 27.1676 \\ \mathrm{H} & -2.5798 & -3.1147 & 27.5672 \\ \mathrm{C} & -2.4877 & -0.2975 & 29.654 \\ \mathrm{H} & -3.2126 & 0.1388 & 30.3786 \\ \mathrm{H} & -0.7324 & 0.2203 & 30.8495 \\ \mathrm{C} & -4.2136 & -1.8618 & 29.1091 \\ \mathrm{~N} & -4.6906 & -2.9268 & 28.461 \\ \mathrm{C} & -5.9468 & -3.3422 & 28.6352 \\ \mathrm{H} & -18.5027 & -12.4937 & 25.7808 \\ \mathrm{H} & -18.4717 & -12.4787 & 23.9808 \\ \mathrm{H} & -17.7748 & -14.6886 & 22.7402 \\ \mathrm{H} & -17.7424 & -14.851 & 25.8386 \\ \mathrm{~N} & -6.7317 & -2.6675 & 29.4779 \\ \mathrm{C} & -6.2859 & -1.5994 & 30.1425 \\ \mathrm{~N} & -7.0416 & -0.9717 & 30.9408 \\ \mathrm{~N} & -5.0247 & -1.2097 & 29.945 \\ \mathrm{H} & -1.4366 & -1.3947 & 31.2088 \\ \mathrm{H} & 0.7235 & -1.6474 & 29.9717 \\ \mathrm{H} & -1.0563 & -3.3469 & 29.5214 \\ \mathrm{H} & -5.4679 & 0.4217 & 31.5065 \\ \mathrm{H} & -7.1214 & 1.0933 & 31.3022 \\ \mathrm{H} & 0.1099 & -0.6136 & 28.6377 \\ \mathrm{H} & -6.5265 & 0.9507 & 33.7382 \\ \mathrm{H} & -8.702 & -2.3419 & 30.6189 \\ \mathrm{H} & -9.0973 & -0.5951 & 30.7594 \\ \mathrm{H} & -14.6094 & -12.9119 & 23.9232 \\ \mathrm{H} & -16.0119 & -12.7427 & 22.8078 \\ \mathrm{H} & -15.301 & -15.1306 & 22.9472 \\ \mathrm{H} & -15.4382 & -15.0995 & 24.7391 \\ \mathrm{H} & -8.0982 & -2.4577 & 33.0304\end{array}$




\begin{tabular}{|c|c|c|c|}
\hline & & & \\
\hline & -2.2754 & 0.4817 & \\
\hline & -9.1661 & -9.5103 & \\
\hline & -8.3721 & & \\
\hline & -7.10 & & \\
\hline & -2.4 & & \\
\hline & & & \\
\hline & & & \\
\hline & -7.3925 & -8.0788 & \\
\hline & -8.6 & & \\
\hline & -8. & & \\
\hline & & & \\
\hline & -6.3 & & \\
\hline & -4.9 & & \\
\hline & -4.9 & & \\
\hline & -4.3 & & \\
\hline & -4.8052 & & \\
\hline & -4. & & 22 \\
\hline & -3.4 & & \\
\hline & -5.0848 & & \\
\hline & & & \\
\hline & & & \\
\hline & & & \\
\hline & -6.9705 & & \\
\hline & -7. & & \\
\hline & -7.6 & & \\
\hline & $-4.4 \varepsilon$ & & \\
\hline & -3.6 & & \\
\hline & -3.2 & & 5722 \\
\hline & 3.7981 & & \\
\hline & & & \\
\hline & -4.4 & & \\
\hline & -4.8 & & 21 \\
\hline & & & \\
\hline & & & \\
\hline & -0.6945 & & \\
\hline & & -12.5 & \\
\hline & -2.7636 & & \\
\hline
\end{tabular}




$\begin{array}{llll}\text { C } & -1.9017 & -10.5093 & 19.1507 \\ \text { H } & -2.2385 & -9.8581 & 18.3102 \\ \text { H } & -2.295 & -10.0587 & 20.0911 \\ \text { C } & -0.3641 & -10.5992 & 19.18 \\ \text { H } & 0.0742 & -9.65 & 18.7884 \\ \text { H } & -0.0314 & -10.7311 & 20.2368 \\ \text { H } & -1.9547 & -13.6506 & 17.8175 \\ \text { C } & 3.6613 & -11.2317 & 18.9076 \\ \text { N } & 4.1324 & -12.2651 & 18.2037 \\ \text { C } & 3.3238 & -13.1031 & 17.5532 \\ \text { N } & 2.0084 & -12.8882 & 17.6269 \\ \text { C } & 1.5005 & -11.8725 & 18.3318 \\ \text { N } & 2.337 & -11.0484 & 18.9747 \\ \text { H } & 4.9706 & -17.629 & 16.5332 \\ \text { C } & 3.3232 & -16.4409 & 16.4908 \\ \text { H } & 3.0576 & -16.678 & 17.5484 \\ \text { H } & 2.7721 & -17.1581 & 15.8387 \\ \text { C } & 2.9145 & -14.9999 & 16.1538 \\ \text { H } & 3.0695 & -14.8059 & 15.0666 \\ \text { C } & 5.2577 & -14.2933 & 16.7967 \\ \text { H } & 5.5588 & -14.0533 & 15.7503 \\ \text { H } & 5.8457 & -13.6389 & 17.4806 \\ \text { C } & 5.5554 & -15.7682 & 17.1005 \\ \text { H } & 6.6268 & -15.9941 & 16.8898 \\ \text { H } & 5.3743 & -15.9818 & 18.1808 \\ \text { H } & 1.8345 & -14.8459 & 16.3809\end{array}$




$$
\begin{array}{llll}
\text { Parameters of } & \mathbf{G}_{\mathbf{4}}-\mathbf{N} \sim \mathbf{N}-\mathbf{G}_{4} \text { (R=piperidine) } \\
\text { C } & 15.5581 & -27.0366 & 5.8586 \\
\mathrm{C} & 16.8904 & -26.4448 & 5.3743 \\
\mathrm{~N} & 12.9148 & -21.0832 & 7.0004 \\
\mathrm{H} & 16.0513 & -24.626 & 4.5227 \\
\mathrm{~N} & 15.1826 & -24.9857 & 7.1172 \\
\mathrm{H} & 9.7164 & -28.1393 & 7.7197 \\
\mathrm{C} & 8.3984 & -26.3901 & 7.5943 \\
\mathrm{~N} & 12.7413 & -18.4707 & 5.9754 \\
\mathrm{C} & 16.8122 & -24.9129 & 5.2872 \\
\mathrm{C} & 11.6356 & -20.3366 & 7.131 \\
\mathrm{H} & 11.6603 & -19.821 & 8.1171 \\
\mathrm{H} & 10.7423 & -21.0021 & 7.1137 \\
\mathrm{C} & 11.5026 & -19.289 & 6.0135 \\
\mathrm{H} & 10.5752 & -18.6894 & 6.1578 \\
\mathrm{H} & 11.413 & -19.7954 & 5.024 \\
\mathrm{C} & 10.7951 & -26.3623 & 8.4023 \\
\mathrm{C} & 9.7816 & -27.0513 & 7.4779 \\
\mathrm{C} & 14.0119 & -19.1931 & 5.731 \\
\mathrm{H} & 13.9362 & -19.6216 & 4.7043 \\
\mathrm{H} & 14.9002 & -18.5202 & 5.7455 \\
\mathrm{C} & 14.1779 & -20.3355 & 6.7506 \\
\mathrm{H} & 15.0133 & -20.9984 & 6.4282 \\
\mathrm{C} & 23.7599 & -13.2529 & 6.437 \\
\mathrm{C} & 9.4839 & -24.1912 & 8.2174 \\
\mathrm{C} & 8.472 & -24.8842 & 7.2937 \\
\mathrm{H} & 14.4644 & -19.896 & 7.7316 \\
\mathrm{C} & 19.6678 & -7.1943 & 8.7375 \\
\mathrm{C} & 18.3034 & -6.5562 & 9.0415 \\
\mathrm{~N} & 16.9554 & -13.3527 & 8.131 \\
\mathrm{C} & 16.4152 & -24.3143 & 6.6435 \\
\mathrm{H} & 17.21 & -24.5141 & 7.3982 \\
\mathrm{H} & 16.3114 & -23.2089 & 6.5573 \\
\mathrm{C} & 15.1948 & -26.4619 & 7.2356 \\
\mathrm{H} & 16.1774 & -7.0768 & 9.0589 \\
\mathrm{H} & 14.2281 & -26.888 & 7.5877 \\
\mathrm{~N} & 18.7092 & -9.3729 & 9.3522 \\
\mathrm{H} & 22.8639 & -11.2914 & 6.1385 \\
& & &
\end{array}
$$




$\begin{array}{llll}\mathrm{H} & 14.751 & -26.7866 & 5.1293 \\ \mathrm{C} & 14.1151 & -24.3331 & 7.3054 \\ \mathrm{~N} & 14.0796 & -23.0155 & 7.0995 \\ \mathrm{H} & 17.1587 & -26.87 & 4.3774 \\ \mathrm{C} & 12.9407 & -22.3303 & 7.2136 \\ \mathrm{~N} & 14.8004 & -14.4827 & 6.7299 \\ \mathrm{~N} & 11.8292 & -22.9846 & 7.5583 \\ \mathrm{C} & 11.8396 & -24.2962 & 7.8031 \\ \mathrm{~N} & 10.7802 & -24.9054 & 8.1335 \\ \mathrm{~N} & 12.9919 & -24.9556 & 7.669 \\ \mathrm{C} & 17.1554 & -7.5481 & 8.7979 \\ \mathrm{H} & 15.6229 & -28.1489 & 5.8975 \\ \mathrm{C} & 17.0917 & -14.756 & 7.6578 \\ \mathrm{H} & 16.7951 & -15.4037 & 8.5145 \\ \mathrm{H} & 18.1386 & -15.0227 & 7.3855 \\ \mathrm{C} & 16.1534 & -15.0361 & 6.4682 \\ \mathrm{H} & 16.1373 & -16.1264 & 6.2384 \\ \mathrm{H} & 16.5385 & -14.5073 & 5.5652 \\ \mathrm{C} & 22.6253 & -11.866 & 8.2215 \\ \mathrm{C} & 23.4457 & -11.8298 & 6.9245 \\ \mathrm{C} & 14.7094 & -13.0591 & 7.1492 \\ \mathrm{H} & 15.094 & -12.4451 & 6.3014 \\ \mathrm{H} & 13.6693 & -12.7203 & 7.3587 \\ \mathrm{C} & 15.5863 & -12.8379 & 8.3925 \\ \mathrm{H} & 15.566 & -11.7619 & 8.6795 \\ \mathrm{H} & 17.6961 & -26.7355 & 6.0886 \\ \mathrm{H} & 17.7889 & -24.4919 & 4.9511 \\ \mathrm{H} & 7.6828 & -26.8835 & 6.8937 \\ \mathrm{C} & 21.6465 & -14.1101 & 7.5322 \\ \mathrm{C} & 22.4718 & -14.0671 & 6.2384 \\ \mathrm{H} & 15.1782 & -13.4227 & 9.2503 \\ \mathrm{H} & 17.3548 & -8.8323 & 9.6152 \\ \mathrm{H} & 19.5409 & -8.6054 & 10.7055 \\ \mathrm{H} & -9.5573 & 9.3875 \\ \mathrm{H} & -8.475 & 9.5599 \\ \mathrm{H} & -10.5603 & 8.9437\end{array}$




$$
\begin{aligned}
& \text { N } \quad 17.8208 \quad-11.3615 \quad 8.7452 \\
& \text { C } \quad 17.9679 \quad-12.61548 .3118 \\
& \text { H } \quad 11.8008 \quad-26.82658 .2834 \\
& \text { H } 10.4998 \quad-26.492 \quad 9.4692 \\
& \text { H } \quad 7.9969 \quad-26.5387 \quad 8.624 \\
& \text { H } \quad 10.1389-26.97236 .4234 \\
& \text { N } \quad 19.2005 \quad-13.0684 \quad 8.0749 \\
& \text { C } \quad 20.2713-12.2923 \quad 8.26 \\
& \text { N } \quad 21.436 \quad-12.72848 .0247 \\
& \text { N } \quad 20.0883 \quad-11.04358 .6956 \\
& \begin{array}{llll}
\mathrm{H} & 19.7224 & -7.445 & 7.6511
\end{array} \\
& \text { H } \quad 18.1599 \quad-5.647 \quad 8.4096 \\
& \text { H } \quad 17.1198 \quad-7.8081 \quad 7.7129 \\
& \text { H } \quad 22.3695 \quad-10.8283 \quad 8.5353 \\
& \text { H } \quad 23.2262 \quad-12.3228 \quad 9.0423 \\
& \text { H } 18.2824 \quad-6.2194 \quad 10.104 \\
& \text { H } 24.3918-11.2593 \quad 7.0857 \\
& \text { H } \quad 20.6957-14.66327 .3562 \\
& \text { H } \quad 22.2073 \quad-14.65648 .3263 \\
& \text { H } \quad 9.561 \quad-23.10917 .9647 \\
& \begin{array}{llll}
\mathrm{H} & 9.1291 & -24.2599 & 9.2708
\end{array} \\
& \text { H } 7.4643 \quad-24.41847 .4138 \\
& \text { H } \quad 8.7838 \quad-24.73516 .2325 \\
& \text { H } \quad 21.8611-13.59775 .4304 \\
& \text { H } \quad 22.7187 \quad-15.10445 .9073 \\
& \text { H } \quad 19.9021 \quad-8.2303 \quad 10.6475 \\
& \begin{array}{llll}
\text { C } & 12.714 & -17.2329 & 6.2347
\end{array} \\
& \begin{array}{llll}
\mathrm{N} & 11.5404 & -16.6558 & 6.4961
\end{array} \\
& \text { C } \quad 11.452 \quad-15.36836 .8256 \\
& \text { N } \quad 5.8534 \quad-19.673413 .4688 \\
& \begin{array}{llll}
\mathrm{N} & 12.5718 & -14.6507 & 6.8959
\end{array} \\
& \text { H } \quad 15.9711 \quad-26.72457 .9903 \\
& \text { C } \quad 13.7609-15.19736 .6313 \\
& \text { N } \quad 13.8233 \quad-16.48996 .2957 \\
& \text { H } 24.3309-13.20725 .4785 \\
& \text { H } \quad 24.4128 \quad-13.76557 .183 \\
& \text { N } \quad 10.3318 \quad-14.86797 .1285 \\
& \begin{array}{llll}
\mathrm{N} & 9.0622 & -14.8002 & 9.5643
\end{array}
\end{aligned}
$$




$\begin{array}{llll}\mathrm{C} & 10.2244 & -13.5112 & 7.7091 \\ \mathrm{H} & 9.3518 & -13.0145 & 7.2243 \\ \mathrm{H} & 11.1167 & -12.8759 & 7.5027 \\ \mathrm{C} & 9.9781 & -13.6743 & 9.2201 \\ \mathrm{H} & 9.6102 & -12.7113 & 9.646 \\ \mathrm{H} & 10.9561 & -13.9214 & 9.6968 \\ \mathrm{C} & 8.9061 & -16.0104 & 8.7053 \\ \mathrm{H} & 9.6705 & -16.7459 & 9.0429 \\ \mathrm{H} & 7.8947 & -16.4645 & 8.8288 \\ \mathrm{C} & 9.1272 & -15.7206 & 7.2137 \\ \mathrm{H} & 9.2045 & -16.6575 & 6.615 \\ \mathrm{H} & 8.2739 & -15.1385 & 6.7937 \\ \mathrm{C} & 15.8942 & 0.5706 & 17.6571 \\ \mathrm{C} & 14.7358 & 1.1489 & 18.4848 \\ \mathrm{~N} & 12.4097 & -5.0632 & 16.1683 \\ \mathrm{H} & 13.1327 & 0.4331 & 17.1985 \\ \mathrm{~N} & 14.8415 & -1.6467 & 17.8664 \\ \mathrm{H} & 19.7187 & -3.3268 & 13.9343 \\ \mathrm{C} & 18.74 & -5.0731 & 13.0498 \\ \mathrm{~N} & 10.5642 & -7.048 & 16.9109 \\ \mathrm{C} & 13.4456 & 0.3435 & 18.2662 \\ \mathrm{C} & 12.2984 & -6.2692 & 15.3094 \\ \mathrm{H} & 13.2549 & -6.5487 & 14.8121 \\ \mathrm{H} & 11.5652 & -6.0248 & 14.5056 \\ \mathrm{C} & 11.7755 & -7.4476 & 16.1449 \\ \mathrm{H} & 12.5454 & -7.7352 & 16.8989 \\ \mathrm{H} & 11.6123 & -8.3283 & 15.4827 \\ \mathrm{C} & 18.087 & -3.9771 & 15.2353 \\ \mathrm{C} & 18.6926 & -3.7573 & 13.8417 \\ \mathrm{C} & 10.6354 & -5.8144 & 17.7367 \\ \mathrm{H} & 9.6502 & -5.5336 & 18.1752 \\ \mathrm{H} & 11.3323 & -6.039 & 18.578 \\ \mathrm{C} & 11.1966 & -4.6382 & 16.914 \\ \mathrm{H} & 10.4465 & -4.3299 & 16.1487 \\ \mathrm{H} & -2.3197 & 23.8625 \\ \mathrm{H} & -3.7613 & 17.5767\end{array}$




$\begin{array}{llll}\mathrm{C} & -2.5438 & -8.6682 & 21.1901 \\ \mathrm{C} & -2.5063 & -9.9721 & 20.3812 \\ \mathrm{~N} & 3.8329 & -6.965 & 19.0573 \\ \mathrm{C} & 13.6614 & -1.1382 & 18.6049 \\ \mathrm{H} & 13.8843 & -1.2532 & 19.6916 \\ \mathrm{H} & 12.7303 & -1.7121 & 18.3934 \\ \mathrm{C} & 16.1211 & -0.9097 & 17.9946 \\ \mathrm{H} & -1.4908 & -10.7837 & 18.6217 \\ \mathrm{H} & 16.9319 & -1.3222 & 17.3517 \\ \mathrm{~N} & -0.1012 & -8.428 & 20.7313 \\ \mathrm{H} & -0.1837 & -3.0554 & 22.7247 \\ \mathrm{H} & 15.654 & 0.6658 & 16.5712 \\ \mathrm{C} & 14.7592 & -2.6843 & 17.1458 \\ \mathrm{~N} & 13.6055 & -3.3474 & 17.0343 \\ \mathrm{H} & 14.5675 & 2.217 & 18.2059 \\ \mathrm{C} & 13.5052 & -4.439 & 16.2736 \\ \mathrm{~N} & 6.3407 & -7.9897 & 18.3073 \\ \mathrm{~N} & 14.5899 & -4.8677 & 15.6248 \\ \mathrm{C} & 15.7601 & -4.2338 & 15.7205 \\ \mathrm{~N} & 16.782 & -4.6691 & 15.1128 \\ \mathrm{~N} & 15.8253 & -3.1411 & 16.4843 \\ \mathrm{C} & -1.5004 & -9.8464 & 19.2285 \\ \mathrm{H} & 16.8287 & 1.153 & 17.8423 \\ \mathrm{C} & 4.9831 & -6.0257 & 19.0459 \\ \mathrm{H} & 4.9083 & -5.2342 & 19.8265 \\ \mathrm{H} & 4.9644 & -5.5167 & 18.0539 \\ \mathrm{C} & 6.3049 & -6.7995 & 19.1972 \\ \mathrm{H} & 6.3723 & -7.1881 & 20.2385 \\ \mathrm{H} & 7.1674 & -6.1128 & 19.035 \\ \mathrm{C} & 0.8702 & -4.7465 & 23.5923 \\ \mathrm{C} & 0.3558 & -3.3031 & 23.6698 \\ \mathrm{C} & 5.1593 & -8.8951 & 18.2907 \\ \mathrm{H} & 5.2216 & -9.6874 & 17.51 \\ \mathrm{H} & 5.1355 & -9.4033 & 19.2829 \\ \mathrm{C} & 3.8626 & -8.0913 & 18.0909 \\ \mathrm{H} & 3.848 & -7.6433 & 17.0698 \\ \mathrm{H} & 15.0068 & 1.1316 & 19.5673 \\ \mathrm{H} & 12.6196 & 0.7624 & 18.8899\end{array}$




$$
\begin{array}{llll}
\text { H } & 19.167 & -4.8883 & 12.0349 \\
\mathrm{C} & 3.0812 & -3.8988 & 22.6543 \\
\mathrm{C} & 2.5501 & -2.4609 & 22.7311 \\
\mathrm{H} & 2.9801 & -8.7661 & 18.1726 \\
\mathrm{C} & -0.0921 & -9.5513 & 19.762 \\
\mathrm{H} & 0.293 & -10.4449 & 20.3039 \\
\mathrm{H} & 0.5869 & -9.3631 & 18.8986 \\
\mathrm{C} & -1.1594 & -8.348 & 21.7685 \\
\mathrm{H} & -1.197 & -7.3526 & 22.2679 \\
\mathrm{H} & -3.2948 & -8.7459 & 22.0128 \\
\mathrm{C} & 0.8598 & -7.6034 & 20.7496 \\
\mathrm{~N} & 1.8637 & -7.7016 & 19.876 \\
\mathrm{C} & 2.8851 & -6.8426 & 19.8865 \\
\mathrm{H} & 18.0143 & -3.0012 & 15.7678 \\
\mathrm{H} & 18.7503 & -4.6415 & 15.8373 \\
\mathrm{H} & 19.4185 & -5.7953 & 13.5634 \\
\mathrm{H} & 18.0714 & -3.0155 & 13.285 \\
\mathrm{~N} & 2.8964 & -5.8692 & 20.799 \\
\mathrm{C} & 1.916 & -5.7547 & 21.6975 \\
\mathrm{~N} & 1.9464 & -4.8478 & 22.5795 \\
\mathrm{~N} & 0.907 & -6.6271 & 21.6576 \\
\mathrm{H} & -2.868 & -7.8319 & 20.5259 \\
\mathrm{H} & -3.5225 & -10.1997 & 19.9786 \\
\mathrm{H} & -1.8194 & -9.0184 & 18.5515 \\
\mathrm{H} & 0.0196 & -5.4413 & 23.4066 \\
\mathrm{H} & 1.3264 & -5.0338 & 24.5652 \\
\mathrm{H} & -2.2165 & -10.8219 & 21.0433 \\
\mathrm{H} & -0.3761 & -3.2029 & 24.5071 \\
\mathrm{H} & 3.788 & -3.9944 & 21.7985 \\
\mathrm{H} & 3.6454 & -4.1429 & 23.5846 \\
\mathrm{H} & 15.6755 & -6.2994 & 14.1888 \\
\mathrm{H} & 17.2963 & -6.6852 & 14.8628 \\
\mathrm{H} & 17.3861 & -6.653 & 12.3688 \\
\mathrm{H} & 16.6858 & -4.9974 & 12.335 \\
\mathrm{H} & 2.0693 & -2.1954 & 21.7595 \\
\mathrm{H} & 3.3958 & -1.749 & 22.8883 \\
\mathrm{H} & -0.9039 & -9.1023 & 22.5449 \\
\mathrm{C} & 9.5236 & -7.7679 & 16.8937
\end{array}
$$




$\begin{array}{llll}\text { N } & 9.501 & -8.866 & 16.1361 \\ \mathrm{C} & 8.437 & -9.6678 & 16.1111 \\ \mathrm{~N} & 7.0978 & -17.2413 & 12.7601 \\ \mathrm{~N} & 7.3793 & -9.3568 & 16.8599 \\ \mathrm{H} & 16.4511 & -1.0139 & 19.0548 \\ \mathrm{C} & 7.3661 & -8.2561 & 17.6148 \\ \mathrm{~N} & 8.4461 & -7.4687 & 17.6255 \\ \mathrm{H} & 1.1315 & -1.2734 & 23.8923 \\ \mathrm{H} & 2.0145 & -2.5147 & 24.8442 \\ \mathrm{~N} & 8.4301 & -10.7057 & 15.3896 \\ \mathrm{~N} & 8.3275 & -12.977 & 13.6425 \\ \mathrm{C} & 7.274 & -11.6353 & 15.444 \\ \mathrm{H} & 6.4947 & -11.2339 & 14.7538 \\ \mathrm{H} & 6.8552 & -11.691 & 16.4766 \\ \mathrm{C} & 7.6894 & -13.044 & 14.9817 \\ \mathrm{H} & 6.7952 & -13.7104 & 14.9776 \\ \mathrm{H} & 8.4465 & -13.461 & 15.6866 \\ \mathrm{C} & 9.0768 & -11.7601 & 13.2396 \\ \mathrm{H} & 9.95-12.022 & 12.5964 \\ \mathrm{H} & 8.3741 & -11.1168 & 12.6592 \\ \mathrm{C} & 9.5679 & -10.9907 & 14.4794 \\ \mathrm{H} & 10.2942 & -11.619 & 15.0469 \\ \mathrm{H} & 10.0783 & -10.0538 & 14.1547 \\ \mathrm{C} & 8.2283 & -13.9491 & 12.8396 \\ \mathrm{~N} & 7.6353 & -15.0819 & 13.2269 \\ \mathrm{C} & 7.5468 & -16.1176 & 12.3907 \\ \mathrm{~N} & 4.7082 & -26.2532 & 10.9096 \\ \mathrm{~N} & 8.0114 & -15.9781 & 11.15 \\ \mathrm{C} & 8.5983 & -14.8541 & 10.7407 \\ \mathrm{~N} & 8.7301 & -13.847 & 11.6056 \\ \mathrm{C} & 6.0786 & -19.3966 & 12.0604 \\ \mathrm{H} & 5.1418 & -18.9838 & 11.6146 \\ \mathrm{H} & 6.338 & -20.3254 & 11.4995 \\ \mathrm{C} & 7.2264 & -18.4047 & 11.859 \\ \mathrm{H} & 8.1914 & -18.8913 & 12.1309 \\ \mathrm{C} & 6.689 & -17.4569 & 14.162 \\ \mathrm{H} & 7.5849 & -17.8549 & 14.6949 \\ \mathrm{H} & 6.375 & -16.5068 & 14.6543\end{array}$




$$
\begin{array}{llll}
\mathrm{C} & \multicolumn{1}{l}{5.55-18.4835} & 14.2436 \\
\mathrm{H} & 5.4098 & -18.7493 & 15.3185 \\
\mathrm{H} & 4.6024 & -18.0254 & 13.8703 \\
\mathrm{H} & 7.2725 & -18.1179 & 10.7827 \\
\mathrm{C} & -8.2935 & -32.5045 & 7.7402 \\
\mathrm{C} & -9.7589 & -32.6779 & 8.168 \\
\mathrm{~N} & -7.7652 & -26.6484 & 11.04 \\
\mathrm{H} & -9.5796 & -31.7593 & 10.1337 \\
\mathrm{~N} & -8.5141 & -30.0993 & 8.2086 \\
\mathrm{H} & -2.0564 & -30.1861 & 6.9184 \\
\mathrm{C} & -1.558 & -28.4598 & 8.1714 \\
\mathrm{~N} & -8.1713 & -26.0963 & 13.7541 \\
\mathrm{C} & -10.1717 & -31.616 & 9.1993 \\
\mathrm{C} & -6.8023 & -25.754 & 11.7311 \\
\mathrm{H} & -7.1688 & -24.7077 & 11.6109 \\
\mathrm{H} & -5.7719 & -25.7957 & 11.311 \\
\mathrm{C} & -6.7839 & -26.1478 & 13.2172 \\
\mathrm{H} & -6.0608 & -25.4998 & 13.7616 \\
\mathrm{H} & -6.43 & -27.1973 & 13.3191 \\
\mathrm{C} & -3.8899 & -29.0954 & 7.4116 \\
\mathrm{C} & -2.49 & -29.6127 & 7.7726 \\
\mathrm{C} & -9.2207 & -26.8753 & 13.0521 \\
\mathrm{H} & -8.9902 & -27.9428 & 13.2684 \\
\mathrm{H} & -10.2435 & -26.6685 & 13.4434 \\
\mathrm{C} & -9.1684 & -26.6487 & 11.5285 \\
\mathrm{H} & -9.8092 & -27.3928 & 11.0021 \\
\mathrm{C} & -20.0324 & -25.6849 & 16.6622 \\
\mathrm{C} & -3.5274 & -27.1311 & 9.0111 \\
\mathrm{C} & -2.1438 & -27.6916 & 9.3642 \\
\mathrm{H} & -9.5759 & -25.637 & 11.2971 \\
\mathrm{C} & -17.5296 & -20.4335 & 21.9038 \\
\mathrm{C} & -16.3936 & -19.5653 & 22.4654 \\
\mathrm{~N} & -13.5605 & -22.9477 & 16.9526 \\
\mathrm{C} & -9.9215 & -30.2003 & 8.6607 \\
\mathrm{H} & -10.5662 & -30.006 & 7.772 \\
\mathrm{H} & -10.19 & -29.448 & 9.4372 \\
\mathrm{C} & -8.0281 & -31.0867 & 7.2166 \\
\mathrm{H} & -14.2229 & -19.3492 & 22.2926
\end{array}
$$




\begin{tabular}{|c|c|c|c|}
\hline & & & \\
\hline & -16.215 & & \\
\hline & & & \\
\hline & 61 & -32.6 & \\
\hline & & -29 . & \\
\hline & -8.18 & & \\
\hline & & & \\
\hline & & & \\
\hline & & & \\
\hline & -6.0 & & \\
\hline & & & \\
\hline & & & \\
\hline & -6.45 & & \\
\hline & & & \\
\hline & -8.0 & & \\
\hline & & & \\
\hline & & & \\
\hline & & & \\
\hline & & & \\
\hline & & & \\
\hline & & & \\
\hline & & & \\
\hline & & & \\
\hline & & & \\
\hline & & & \\
\hline & & & \\
\hline & & & \\
\hline & -12 & & \\
\hline & & & \\
\hline & & & \\
\hline & & & \\
\hline & -17 & & 15.8 \\
\hline & -18 & & 16.206 \\
\hline & -11.9653 & & 16.719 \\
\hline & & -19. & \\
\hline & & & 20.006 \\
\hline & & & 19.979 \\
\hline & & -20.37 & \\
\hline
\end{tabular}




\begin{tabular}{|c|c|c|c|}
\hline & & & \\
\hline & -18.5109 & & \\
\hline & & & \\
\hline & & & \\
\hline & & & \\
\hline & -4.5637 & -29.96 & \\
\hline & & -28.4 & \\
\hline & & -27.77 & \\
\hline & -2.5728 & -30 . & \\
\hline & -15 . & -23 & \\
\hline & & & \\
\hline & & & \\
\hline & & -22 & \\
\hline & & & \\
\hline & & & \\
\hline & & & \\
\hline & & & \\
\hline & & & \\
\hline & & & \\
\hline & -21 . & & \\
\hline & & & \\
\hline & & & \\
\hline & -3.9 & -26.6 & \\
\hline & & -26 . & \\
\hline & & & \\
\hline & -2.2 & & \\
\hline & & & \\
\hline & -18 & -26.653 & \\
\hline & & & \\
\hline & & & \\
\hline & & -24 & \\
\hline & -7.635 & -23. & 16. \\
\hline & & & \\
\hline & -8.8 & -23.7489 & \\
\hline & & -30.9 & \\
\hline & -9.9069 & -24 & 260 \\
\hline & -9.6793 & -25.2189 & 15.23 \\
\hline & -20.5109 & -26.6464 & \\
\hline
\end{tabular}




\begin{tabular}{|c|c|c|}
\hline & & -2 \\
\hline & & \\
\hline & & -21.3469 16.983 \\
\hline & 7 & 231 \\
\hline & & \\
\hline & -7.8 & \\
\hline & -6.1 & \\
\hline & & 871 \\
\hline & & \\
\hline & -4.6 & \\
\hline & & \\
\hline & & \\
\hline & & \\
\hline & & 3215 \\
\hline & & \\
\hline & & \\
\hline & & \\
\hline & & \\
\hline & & \\
\hline & & \\
\hline & & -15.8 \\
\hline & & \\
\hline & & 9722 \\
\hline & & \\
\hline & -8.7084 & -13 \\
\hline & & 2421 \\
\hline & & $\begin{array}{ll}-13.624 & 21\end{array}$ \\
\hline & -7.3239 & -13.6517 \\
\hline & -7.0 & -13 \\
\hline & -7.3674 & 93722 \\
\hline & & -14 \\
\hline & & -15 \\
\hline & -6.6 & 99923. \\
\hline & & -11.672824 .207 \\
\hline & -6.895 & -10.969922 .914 \\
\hline & -7.9306 & -12.351424 \\
\hline & -7.6645 & -13.223824 .895 \\
\hline & 1.2376 & $\begin{array}{ll}-5.9971 & 29.531\end{array}$ \\
\hline
\end{tabular}




$$
\begin{array}{llll}
\mathrm{C} & -13.0828 & -14.2479 & 21.2765 \\
\mathrm{C} & -13.6304 & -15.6018 & 20.803 \\
\mathrm{H} & -8.2995 & -11.5168 & 24.8946 \\
\mathrm{C} & 6.0456 & -7.8155 & 24.099 \\
\mathrm{C} & 6.5163 & -8.8353 & 23.0483 \\
\mathrm{~N} & 0.1155 & -10.6888 & 24.4765 \\
\mathrm{C} & -10.744 & -12.4711 & 27.7528 \\
\mathrm{H} & -11.0352 & -11.4535 & 28.1038 \\
\mathrm{H} & -9.7557 & -12.3793 & 27.2464 \\
\mathrm{C} & -13.1627 & -13.1957 & 27.4263 \\
\mathrm{H} & 5.7918 & -10.5781 & 21.9357 \\
\mathrm{H} & -13.8845 & -13.6152 & 26.6889 \\
\mathrm{~N} & 3.7564 & -8.2674 & 23.4087 \\
\mathrm{H} & 3.1092 & -6.7795 & 28.7385 \\
\mathrm{H} & -12.7193 & -15.1548 & 28.2577 \\
\mathrm{C} & -11.605 & -13.1057 & 25.6136 \\
\mathrm{~N} & -10.3984 & -12.8658 & 25.0959 \\
\mathrm{H} & -11.8916 & -14.3434 & 30.4756 \\
\mathrm{C} & -10.1615 & -13.0305 & 23.7928 \\
\mathrm{~N} & -1.895 & -12.4281 & 23.4522 \\
\mathrm{~N} & -11.1533 & -13.4431 & 23.0008 \\
\mathrm{C} & -12.3694 & -13.697 & 23.4904 \\
\mathrm{~N} & -13.3067 & -14.0866 & 22.7333 \\
\mathrm{~N} & -12.5767 & -13.5202 & 24.7974 \\
\mathrm{C} & 5.4446 & -9.8972 & 22.7497 \\
\mathrm{H} & -14.0229 & -14.2703 & 29.1242 \\
\mathrm{C} & -1.0865 & -10.9471 & 25.3059 \\
\mathrm{H} & -1.3743 & -10.0698 & 25.9298 \\
\mathrm{H} & -0.824 & -11.7846 & 25.9938 \\
\mathrm{C} & -2.2701 & -11.3602 & 24.4144 \\
\mathrm{H} & -2.5966 & -10.4767 & 23.8169 \\
\mathrm{H} & -3.1152 & -11.7025 & 25.0577 \\
\mathrm{C} & 2.009 & -5.8148 & 27.1306 \\
\mathrm{C} & 2.4539 & -5.8869 & 28.598 \\
\mathrm{C} & -0.4739 & -12.8318 & 23.3067 \\
\mathrm{H} & -0.2554 & -13.5075 & 24.1665 \\
\mathrm{H} & -0.2875 & -13.4026 & 22.3677 \\
\mathrm{C} & 0.4648 & -11.6144 & 23.3696
\end{array}
$$




\begin{tabular}{|c|c|c|c|}
\hline & & & \\
\hline & & & \\
\hline & -9.8862 & & \\
\hline & 703 & & \\
\hline & & & \\
\hline & .3706 & & \\
\hline & 34 & & \\
\hline & & & \\
\hline & & & \\
\hline & 3.32 & & \\
\hline & & & \\
\hline & & & \\
\hline & 6.82 & & \\
\hline & & & \\
\hline & & & \\
\hline & 0.8 & & \\
\hline & & & \\
\hline & & & \\
\hline & & & \\
\hline & -14.602 & & \\
\hline & 0.57 & & \\
\hline & & & \\
\hline & & & \\
\hline & 2.4385 & & \\
\hline & 5.9 & & \\
\hline & & & \\
\hline & & & \\
\hline & 2.9011 & & \\
\hline & 1.4 & & \\
\hline & & & \\
\hline & & & \\
\hline & -0.6639 & -8.0 & \\
\hline & -0.7411 & & 27 \\
\hline & -12.0102 & & 20.98 \\
\hline & & & \\
\hline & & & \\
\hline & -13.007 & & 21.23 \\
\hline & 0.9606 & -8.1471 & \\
\hline
\end{tabular}




$\begin{array}{llll}\mathrm{H} & -0.5214 & -7.2636 & 29.8403 \\ \mathrm{H} & 4.836 & -6.587 & 22.7485 \\ \mathrm{C} & -5.0647 & -13.2337 & 22.3132 \\ \mathrm{~N} & -4.7584 & -14.1972 & 21.4443 \\ \mathrm{C} & -3.4964 & -14.5651 & 21.2177 \\ \mathrm{~N} & -0.2777 & -20.9814 & 17.104 \\ \mathrm{~N} & -2.5215 & -13.9563 & 21.8973 \\ \mathrm{H} & -13.5641 & -12.2113 & 27.7631 \\ \mathrm{C} & -2.8057 & -12.995 & 22.781 \\ \mathrm{~N} & -4.0767 & -12.6309 & 22.9752 \\ \mathrm{H} & 1.5795 & -6.0764 & 30.591 \\ \mathrm{H} & 0.6244 & -5.0679 & 29.4533 \\ \mathrm{~N} & -3.2607 & -15.4782 & 20.3743 \\ \mathrm{~N} & -2.8831 & -18.1337 & 19.5346 \\ \mathrm{C} & -1.8753 & -15.9004 & 20.0436 \\ \mathrm{H} & -1.1094 & -15.3939 & 20.6748 \\ \mathrm{H} & -1.7015 & -15.584 & 18.9886 \\ \mathrm{C} & -1.7215 & -17.4307 & 20.1386 \\ \mathrm{H} & -1.7124 & -17.7241 & 21.2146 \\ \mathrm{H} & -0.7495 & -17.7486 & 19.6957 \\ \mathrm{C} & -4.2458 & -17.6864 & 19.9292 \\ \mathrm{H} & -5.0643 & -18.2176 & 19.3925 \\ \mathrm{H} & -4.3604 & -17.9054 & 21.0168 \\ \mathrm{C} & -4.3771 & -16.1746 & 19.6833 \\ \mathrm{H} & -4.279 & -15.9541 & 18.5945 \\ \mathrm{H} & -5.3863 & -15.8301 & 20.0049 \\ \mathrm{C} & -2.7312 & -19.0958 & 18.7263 \\ \mathrm{~N} & -1.5221 & -19.5025 & 18.3234 \\ \mathrm{C} & -1.4 & -20.5342 & 17.4818 \\ \mathrm{~N} & 4.1988 & -23.7801 & 12.1729 \\ \mathrm{~N} & -2.5057 & -21.1362 & 17.0424 \\ \mathrm{C} & -3.7219 & -20.7485 & 17.4214 \\ \mathrm{~N} & -3.8162 & -19.7294 & 18.2732 \\ \mathrm{C} & 0.8201 & -22.1016 & 15.1832 \\ \mathrm{H} & 0.9548 & -23.1134 & 14.7332 \\ \mathrm{H} & 0.4156 & -21.4242 & 14.3932 \\ \mathrm{C} & -0.1931 & -22.2329 & 16.322 \\ \mathrm{H} & -1.1703 & -22.5615 & 15.9019\end{array}$




\begin{tabular}{|c|c|c|}
\hline & & \\
\hline & 0.8263 & -19.3276 17.941 \\
\hline & & \\
\hline & & 171 \\
\hline & & \\
\hline & & \\
\hline & & \\
\hline & & \\
\hline & & \\
\hline & & -22 . \\
\hline & & \\
\hline & & \\
\hline & & \\
\hline & & -20 \\
\hline & & -26 \\
\hline & & \\
\hline & & -26 \\
\hline & & \\
\hline & & 6711 \\
\hline & & -23.89 \\
\hline & & -23 \\
\hline & & 9911 \\
\hline & & -25 \\
\hline & & -25 \\
\hline & & -25 \\
\hline & & 2413 \\
\hline & & -15.856 \\
\hline & 15.239 & -22 \\
\hline & & \\
\hline & & -18.6 \\
\hline & 7.99 & -21. \\
\hline & & -23.2 \\
\hline & 17.4914 & 27910 \\
\hline & 14.6 & -16.775410 .643 \\
\hline & & -24.1 \\
\hline & 14.9604 & -24.7669 \\
\hline & & -24.295111 .7685 \\
\hline & 16.3748 & -24.537611 .835 \\
\hline
\end{tabular}




$\begin{array}{llll}\text { H } & 16.3057 & -25.5751 & 12.233 \\ \mathrm{H} & 16.5455 & -23.8766 & 12.7169 \\ \mathrm{C} & 9.6169 & -21.5561 & 11.6261 \\ \mathrm{C} & 9.0004 & -21.7343 & 13.021 \\ \mathrm{C} & 17.7792 & -22.9275 & 10.4995 \\ \mathrm{H} & 18.1027 & -22.4064 & 11.4305 \\ \mathrm{H} & 18.6141 & -22.8515 & 9.7645 \\ \mathrm{C} & 16.4894 & -22.2704 & 9.9568 \\ \mathrm{H} & 16.5915 & -21.1604 & 9.9661 \\ \mathrm{C} & 26.5889 & -21.5769 & 3.2811 \\ \mathrm{C} & 10.8623 & -23.7549 & 11.9308 \\ \mathrm{C} & 10.2363 & -23.92 & 13.3219 \\ \mathrm{H} & 16.3669 & -22.5868 & 8.8962 \\ \mathrm{C} & 26.2526 & -29.137 & 1.5903 \\ \mathrm{C} & 25.5751 & -30.5057 & 1.7568 \\ \mathrm{~N} & 21.4928 & -25.9821 & 5.2688 \\ \mathrm{C} & 14.4706 & -18.0151 & 9.7634 \\ \mathrm{H} & 14.3655 & -17.7012 & 8.6993 \\ \mathrm{H} & 15.3547 & -18.6894 & 9.833 \\ \mathrm{C} & 11.9764 & -17.8509 & 10.194 \\ \mathrm{H} & 23.9761 & -31.4486 & 2.9159 \\ \mathrm{H} & 11.0767 & -18.4076 & 10.5428 \\ \mathrm{~N} & 24.1317 & -28.0793 & 2.2585 \\ \mathrm{H} & 27.3645 & -23.606 & 3.4189 \\ \mathrm{H} & 12.335 & -16.9419 & 12.1357 \\ \mathrm{C} & 13.1672 & -19.9112 & 10.4413 \\ \mathrm{~N} & 14.2679 & -20.6668 & 10.36 \\ \mathrm{H} & 13.6274 & -14.9703 & 11.2733 \\ \mathrm{C} & 14.2327 & -21.9556 & 10.705 \\ \mathrm{~N} & 20.2345 & -26.2559 & 7.7876 \\ \mathrm{~N} & 13.0772 & -22.4737 & 11.1208 \\ \mathrm{C} & 11.9549 & -21.7613 & 11.1809 \\ \mathrm{~N} & 10.882 & -22.3191 & 11.5556 \\ \mathrm{~N} & 12.0197 & -20.4734 & 10.8367 \\ \mathrm{C} & 24.4798 & -30.4555 & 2.833 \\ \mathrm{H} & 11.3112 & -15.9506 & 11.0448 \\ \mathrm{C} & 21.0214 & -24.7532 & 5.958 \\ \mathrm{H} & 20.0081 & -24.5377 & 5.5482\end{array}$




$$
\begin{array}{llll}
\mathrm{H} & 21.6611 & -23.8652 & 5.7499 \\
\mathrm{C} & 20.9123 & -24.9712 & 7.4775 \\
\mathrm{H} & 20.4114 & -24.0937 & 7.9477 \\
\mathrm{H} & 21.9382 & -25.049 & 7.9081 \\
\mathrm{C} & 25.7188 & -23.5764 & 1.9988 \\
\mathrm{C} & 26.953 & -22.9237 & 2.6371 \\
\mathrm{C} & 20.7117 & -27.4795 & 7.0901 \\
\mathrm{H} & 21.7334 & -27.6865 & 7.4868 \\
\mathrm{H} & 20.0822 & -28.3755 & 7.2953 \\
\mathrm{C} & 20.7968 & -27.2582 & 5.5702 \\
\mathrm{H} & 21.2768 & -28.1429 & 5.0928 \\
\mathrm{H} & 13.3017 & -15.4685 & 9.5805 \\
\mathrm{H} & 15.5919 & -16.2163 & 10.3029 \\
\mathrm{H} & 8.4336 & -23.3268 & 14.4057 \\
\mathrm{C} & 24.2305 & -22.3941 & 3.6904 \\
\mathrm{C} & 25.4712 & -21.7469 & 4.3217 \\
\mathrm{H} & 19.7726 & -27.1652 & 5.1425 \\
\mathrm{C} & 23.4444 & -29.3664 & 2.5194 \\
\mathrm{H} & 22.8858 & -29.6275 & 1.5907 \\
\mathrm{H} & 22.7008 & -29.306 & 3.3466 \\
\mathrm{C} & 25.2182 & -28.0548 & 1.2509 \\
\mathrm{H} & 25.7223 & -27.0644 & 1.1736 \\
\mathrm{H} & 27.0299 & -29.1871 & 0.7903 \\
\mathrm{C} & 23.7902 & -27.0241 & 2.8689 \\
\mathrm{~N} & 22.8102 & -27.0455 & 3.7746 \\
\mathrm{C} & 22.4302 & -25.9406 & 4.4199 \\
\mathrm{H} & 9.7573 & -20.4752 & 11.399 \\
\mathrm{H} & 8.9357 & -21.977 & 10.8497 \\
\mathrm{H} & 8.1669 & -23.7193 & 12.6748 \\
\mathrm{H} & 9.6447 & -21.2232 & 13.7755 \\
\mathrm{~N} & 23.0521 & -24.793 & 4.1427 \\
\mathrm{C} & 24.04 & -24.7372 & 3.2467 \\
\mathrm{~N} & 24.6245 & -23.6426 & 2.996 \\
\mathrm{~N} & 24.3952 & -25.8607 & 2.6191 \\
\mathrm{H} & 26.7711 & -28.8641 & 2.5404 \\
\mathrm{H} & 26.3361 & -31.277 & 2.0262 \\
\mathrm{H} & 24.9494 & -30.2374 & 3.8218 \\
\mathrm{H} & 25.993 & -24.5719 & 1.581
\end{array}
$$




$\begin{array}{llll}\mathrm{H} & 25.3494 & -22.9503 & 1.1532 \\ \mathrm{H} & 25.129 & -30.8154 & 0.7831 \\ \mathrm{H} & 27.7505 & -22.7806 & 1.8688 \\ \mathrm{H} & 23.4508 & -22.5524 & 4.4701 \\ \mathrm{H} & 23.797 & -21.7164 & 2.9181 \\ \mathrm{H} & 11.8669 & -24.2341 & 11.9122 \\ \mathrm{H} & 10.2379 & -24.2846 & 11.1769 \\ \mathrm{H} & 10.127 & -25.0038 & 13.5679 \\ \mathrm{H} & 10.9156 & -23.4716 & 14.0857 \\ \mathrm{H} & 25.8386 & -22.3942 & 5.1536 \\ \mathrm{H} & 25.2032 & -20.7577 & 4.7649 \\ \mathrm{H} & 24.7494 & -28.2701 & 0.2622 \\ \mathrm{C} & 17.9897 & -25.3184 & 10.2749 \\ \mathrm{~N} & 17.4876 & -26.5178 & 10.5631 \\ \mathrm{C} & 17.8527 & -27.6175 & 9.9118 \\ \mathrm{~N} & 7.9075 & -29.7863 & 11.0224 \\ \mathrm{~N} & 18.7786 & -27.5058 & 8.9592 \\ \mathrm{H} & 11.7856 & -17.5309 & 9.1442 \\ \mathrm{C} & 19.3318 & -26.3221 & 8.673 \\ \mathrm{~N} & 18.9237 & -25.2266 & 9.3256 \\ \mathrm{H} & 27.4918 & -21.1319 & 3.7643 \\ \mathrm{H} & 26.2557 & -20.8637 & 2.49 \\ \mathrm{~N} & 17.2537 & -28.6992 & 10.1737 \\ \mathrm{~N} & 14.8721 & -29.8077 & 9.2956 \\ \mathrm{C} & 17.4172 & -29.8922 & 9.317 \\ \mathrm{H} & 17.5612 & -30.7682 & 9.9916 \\ \mathrm{H} & 18.3104 & -29.8296 & 8.6529 \\ \mathrm{C} & 16.1169 & -30.0448 & 8.5044 \\ \mathrm{H} & 16.1173 & -31.0426 & 8.0076 \\ \mathrm{H} & 16.1203 & -29.2644 & 7.7119 \\ \mathrm{C} & 14.8256 & -28.7739 & 10.3676 \\ \mathrm{H} & 14.6715 & -27.7916 & 9.8638 \\ \mathrm{H} & 13.9829 & -28.939 & 11.0785 \\ \mathrm{C} & 16.1437 & -28.7342 & 11.1561 \\ \mathrm{H} & 16.1644 & -27.887 & 11.8785 \\ \mathrm{H} & 16.2628 & -29.665 & 11.7586 \\ \mathrm{C} & 24.5934 & -38.9922 & 1.6786 \\ \mathrm{C} & 24.1682 & -40.3659 & 2.2205\end{array}$




$\begin{array}{llll}\mathrm{N} & 18.1719 & -37.108 & 0.6733 \\ \mathrm{H} & 22.4045 & -39.6736 & 3.2922 \\ \mathrm{~N} & 22.3576 & -38.7795 & 0.6697 \\ \mathrm{H} & 24.6142 & -32.8883 & -0.7894 \\ \mathrm{C} & 22.6662 & -31.8971 & -0.9073 \\ \mathrm{~N} & 15.658 & -37.1944 & 2.0033 \\ \mathrm{C} & 22.6562 & -40.4093 & 2.4913 \\ \mathrm{C} & 17.2108 & -36 & 0.4478 \\ \mathrm{H} & 16.6288 & -36.258 & -0.4678 \\ \mathrm{H} & 17.7133 & -35.0216 & 0.2686 \\ \mathrm{C} & 16.2645 & -35.8836 & 1.655 \\ \mathrm{H} & 15.463 & -35.1401 & 1.4299 \\ \mathrm{H} & 16.8529 & -35.5418 & 2.5397 \\ \mathrm{C} & 23.0533 & -34.388 & -1.093 \\ \mathrm{C} & 23.5574 & -33.0733 & -0.4804 \\ \mathrm{C} & 16.16 & -38.4359 & 1.3636 \\ \mathrm{H} & 15.8448 & -39.3532 & 1.9132 \\ \mathrm{H} & 15.7113 & -38.475 & 0.3434 \\ \mathrm{C} & 17.6963 & -38.3901 & 1.2544 \\ \mathrm{H} & 18.1283 & -38.4694 & 2.2804 \\ \mathrm{C} & 11.1559 & -48.7981 & 3.6634 \\ \mathrm{C} & 20.6833 & -33.4491 & -1.11 \\ \mathrm{C} & 21.2075 & -32.144 & -0.4946 \\ \mathrm{H} & 18.0576 & -39.2434 & 0.6324 \\ \mathrm{C} & 4.7547 & -44.5342 & 5.6023 \\ \mathrm{C} & 4.1356 & -43.1502 & 5.8412 \\ \mathrm{~N} & 11.0098 & -41.804 & 4.7543 \\ \mathrm{C} & 21.8638 & -40.0629 & 1.2229 \\ \mathrm{H} & 22.0339 & -40.8401 & 0.4416 \\ \mathrm{H} & 20.7731 & -40.0532 & 1.4505 \\ \mathrm{C} & 23.8093 & -38.6394 & 0.4068 \\ \mathrm{H} & 4.7519 & -41.1302 & 6.4077 \\ \mathrm{H} & 24.0956 & -37.6212 & 0.0574 \\ \mathrm{~N} & 6.7925 & -43.382 & 4.7756 \\ \mathrm{H} & 9.5744 & -48.3373 & 5.0874 \\ \mathrm{H} & 24.3997 & -38.2144 & 2.4554 \\ \mathrm{C} & 21.5596 & -37.8267 & 0.43 \\ \mathrm{~N} & 20.2535 & -37.9587 & 0.6715\end{array}$




\begin{tabular}{|c|c|c|c|}
\hline & & & \\
\hline & 19.403 & -36.9609 & \\
\hline & & & \\
\hline & & & \\
\hline & & & \\
\hline & & & \\
\hline & & & \\
\hline & & & \\
\hline & & & \\
\hline & 73 & & \\
\hline & & & \\
\hline & & -42.08 & \\
\hline & 3.0552 & -40 & \\
\hline & 12.68 & & \\
\hline & & & \\
\hline & & & \\
\hline & 9.68 & -48.474 & \\
\hline & & & \\
\hline & & & \\
\hline & 11.1283 & -38.486 & \\
\hline & 10.4604 & & \\
\hline & 9.74 & & \\
\hline & 24.43 & & \\
\hline & 22.3599 & -41.4 & \\
\hline & & -30 & \\
\hline & 11.6 & & 3.3 \\
\hline & & & \\
\hline & 9.9218 & -40 . & \\
\hline & 6.29 & & 548 \\
\hline & 5.868 & & \\
\hline & & & \\
\hline & 5.8174 & -44. & 4.4 \\
\hline & 6.2982 & -45. & 4.3821 \\
\hline & 3.9599 & -45.2669 & 5.322 \\
\hline & & & \\
\hline & 8.9073 & & \\
\hline & 10.2143 & -42.77 & $4-500$ \\
\hline & 23.6793 & -35.23 & -0.7 \\
\hline
\end{tabular}




$\begin{array}{llll}\mathrm{H} & 23.1542 & -34.352 & -2.203 \\ \mathrm{H} & 22.7259 & -31.7643 & -2.0136 \\ \mathrm{H} & 23.548 & -33.1571 & 0.6327 \\ \mathrm{~N} & 10.6486 & -43.9746 & 4.2045 \\ \mathrm{C} & 9.792 & -44.9755 & 3.9853 \\ \mathrm{~N} & 10.2021 & -46.0981 & 3.5688 \\ \mathrm{~N} & 8.4898 & -44.7611 & 4.1866 \\ \mathrm{H} & 5.2249 & -44.8974 & 6.547 \\ \mathrm{H} & 3.3552 & -43.2109 & 6.6374 \\ \mathrm{H} & 5.6818 & -42.4509 & 7.1995 \\ \mathrm{H} & 8.1953 & -46.9458 & 3.5479 \\ \mathrm{H} & 9.2712 & -47.3442 & 2.1665 \\ \mathrm{H} & 3.6338 & -42.8078 & 4.907 \\ \mathrm{H} & 9.031 & -49.3251 & 3.6862 \\ \mathrm{H} & 12.2897 & -45.4922 & 3.66 \\ \mathrm{H} & 11.7751 & -46.4478 & 2.2263 \\ \mathrm{H} & 19.6428 & -33.6347 & -0.7582 \\ \mathrm{H} & 20.6499 & -33.3579 & -2.221 \\ \mathrm{H} & 20.5668 & -31.2863 & -0.8119 \\ \mathrm{H} & 21.1459 & -32.2093 & 0.6181 \\ \mathrm{H} & 12.0299 & -47.4661 & 5.1457 \\ \mathrm{H} & 13.1346 & -47.8699 & 3.7861 \\ \mathrm{H} & 5.3258 & -44.2125 & 3.5317 \\ \mathrm{C} & 14.7372 & -37.2299 & 2.8697 \\ \mathrm{~N} & 14.363 & -36.0913 & 3.4556 \\ \mathrm{C} & 13.4033 & -36.0736 & 4.3807 \\ \mathrm{~N} & 10.4172 & -30.4336 & 9.7763 \\ \mathrm{~N} & 12.8126 & -37.2203 & 4.7235 \\ \mathrm{H} & 24.0623 & -39.3554 & -0.4098 \\ \mathrm{C} & 13.1778 & -38.3714 & 4.1525 \\ \mathrm{~N} & 14.138 & -38.3689 & 3.2227 \\ \mathrm{H} & 11.4695 & -49.7186 & 4.212 \\ \mathrm{H} & 11.2587 & -49.0149 & 2.5735 \\ \mathrm{H} & 12.0704 & -34.9842 & 4.9298 \\ \mathrm{H} & -32.7188 & 6.6296 \\ \mathrm{H} & 11.9741 & -34.9715 & 5.9311 \\ \mathrm{H} & -35.4565 & 6.8568 \\ \mathrm{H} & -35.5529 & 5.5487\end{array}$




$\begin{array}{llll}\mathrm{C} & 11.5387 & -33.5311 & 6.266 \\ \mathrm{H} & 10.7773 & -33.5475 & 7.0803 \\ \mathrm{H} & 11.0802 & -33.0558 & 5.3672 \\ \mathrm{C} & 14.0127 & -32.9275 & 5.9164 \\ \mathrm{H} & 14.4837 & -31.945 & 5.674 \\ \mathrm{H} & 14.6836 & -33.5045 & 6.5965 \\ \mathrm{C} & 13.8017 & -33.7291 & 4.6203 \\ \mathrm{H} & 13.1697 & -33.1467 & 3.9096 \\ \mathrm{H} & 14.7884 & -33.9153 & 4.1362 \\ \mathrm{C} & 12.6757 & -31.8723 & 7.5674 \\ \mathrm{~N} & 11.534 & -31.5973 & 8.2006 \\ \mathrm{C} & 11.507 & -30.7045 & 9.1937 \\ \mathrm{~N} & 12.6418 & -30.1068 & 9.5646 \\ \mathrm{C} & 13.7958 & -30.3768 & 8.9489 \\ \mathrm{~N} & 13.7947 & -31.2486 & 7.9397 \\ \mathrm{C} & 8.9735 & -28.8076 & 10.9307 \\ \mathrm{H} & 8.8998 & -28.0994 & 11.7895 \\ \mathrm{H} & 8.8395 & -28.2234 & 9.9921 \\ \mathrm{C} & 10.3732 & -29.4391 & 10.8731 \\ \mathrm{H} & 11.1426 & -28.6441 & 10.7317 \\ \mathrm{C} & 9.1752 & -31.1325 & 9.3782 \\ \mathrm{H} & 8.7172 & -30.5709 & 8.5301 \\ \mathrm{H} & 9.3817 & -32.1821 & 9.0622 \\ \mathrm{C} & 8.228 & -31.1303 & 10.5825 \\ \mathrm{H} & 7.323 & -31.7496 & 10.3861 \\ \mathrm{H} & 8.7371 & -31.6411 & 11.4334 \\ \mathrm{H} & 10.5867 & -29.9778 & 11.826 \\ \mathrm{C} & -8.0391 & -25.6664 & 19.8411 \\ \mathrm{C} & -9.4306 & -26.2803 & 20.0536 \\ \mathrm{~N} & -6.7097 & -29.78 & 14.5383 \\ \mathrm{H} & -9.9243 & -26.4906 & 17.9446 \\ \mathrm{~N} & -7.4589 & -27.6444 & 18.4742 \\ \mathrm{H} & -1.3891 & -24.9951 & 17.9307 \\ \mathrm{C} & -0.9411 & -25.9106 & 15.9908 \\ \mathrm{~N} & -7.5818 & -31.2367 & 12.3258 \\ \mathrm{C} & -9.8445 & -27.1381 & 18.8485 \\ \mathrm{C} & -5.783 & -30.0162 & 13.3965 \\ \mathrm{H} & -5.2334 & -30.9651 & 13.6008\end{array}$




\begin{tabular}{|c|c|c|}
\hline & & \\
\hline & & $\begin{array}{ll}-30.1492 & 12.107\end{array}$ \\
\hline & 5.9468 & \\
\hline & 1 & -29 \\
\hline & 35 & \\
\hline & 36 & -25 . \\
\hline & 8 & -31 \\
\hline & & -3 \\
\hline & & \\
\hline & -7.9 & \\
\hline & & \\
\hline & & \\
\hline & -2.6 & -27 \\
\hline & & 981 \\
\hline & & \\
\hline & & \\
\hline & -9.6 & \\
\hline & & -37 \\
\hline & & -2 \\
\hline & -8.7 & -28 \\
\hline & & \\
\hline & & -20 \\
\hline & -8.3 & -43 \\
\hline & -6.00 & \\
\hline & & \\
\hline & & -41 \\
\hline & & -24.5 \\
\hline & -6.714 & -27. \\
\hline & & -28 \\
\hline & & -25. \\
\hline & & \\
\hline & & -35 \\
\hline & & -28 . \\
\hline & & 19916.360 \\
\hline & & -27.1 \\
\hline & & 74717.403 \\
\hline & & $\begin{array}{ll}-43.331 & 10.544\end{array}$ \\
\hline & -7.7406 & -25.0627 \\
\hline
\end{tabular}




$$
\begin{array}{llll}
\mathrm{C} & -9.7058 & -36.6186 & 13.6205 \\
\mathrm{H} & -8.873 & -36.3337 & 14.3055 \\
\mathrm{H} & -10.6407 & -36.6677 & 14.2243 \\
\mathrm{C} & -9.8533 & -35.5373 & 12.5327 \\
\mathrm{H} & -9.9911 & -34.5347 & 12.9991 \\
\mathrm{H} & -10.7572 & -35.7468 & 11.914 \\
\mathrm{C} & -13.0838 & -41.3017 & 16.0112 \\
\mathrm{C} & -14.602 & -41.1473 & 15.8436 \\
\mathrm{C} & -8.3896 & -36.9019 & 10.9763 \\
\mathrm{H} & -9.2721 & -37.1574 & 10.344 \\
\mathrm{H} & -7.5026 & -36.8769 & 10.3029 \\
\mathrm{C} & -8.202 & -37.9741 & 12.0614 \\
\mathrm{H} & -8.0733 & -38.9707 & 11.5807 \\
\mathrm{H} & -9.4222 & -26.911 & 20.974 \\
\mathrm{H} & -10.8521 & -27.5869 & 19.0214 \\
\mathrm{H} & -0.2378 & -25.0993 & 15.6883 \\
\mathrm{C} & -12.8516 & -38.772 & 16.1507 \\
\mathrm{C} & -14.3705 & -38.6279 & 15.9809 \\
\mathrm{H} & -7.2751 & -37.7537 & 12.6412 \\
\mathrm{C} & -8.6852 & -42.4838 & 11.7388 \\
\mathrm{H} & -7.7979 & -42.9546 & 12.2202 \\
\mathrm{H} & -8.3707 & -41.4777 & 11.3785 \\
\mathrm{C} & -10.3616 & -43.6941 & 13.2284 \\
\mathrm{H} & -11.2115 & -43.5419 & 13.9321 \\
\mathrm{H} & -11.2028 & -45.5139 & 12.3594 \\
\mathrm{C} & -10.1198 & -41.3163 & 13.2569 \\
\mathrm{~N} & -9.5402 & -40.1757 & 12.8772 \\
\mathrm{C} & -9.9069 & -38.9991 & 13.3898 \\
\mathrm{H} & -3.6878 & -26.0638 & 18.1432 \\
\mathrm{H} & -2.3564 & -27.2713 & 18.037 \\
\mathrm{H} & -10.8236 & -26.7319 & 16.426 \\
\mathrm{H} & -24.533 & -38.9756 & 14.2991 \\
\mathrm{H} & -40.0971 & 14.7008 \\
\mathrm{H} & -40.0596 & 15.5639 \\
\mathrm{H} & -41.2577 & 14.1739 \\
\mathrm{H} & -43.992 & 11.5324 \\
\mathrm{H} & -10.1258
\end{array}
$$




$\begin{array}{llll}\mathrm{H} & -9.9537 & -42.7821 & 9.9996 \\ \mathrm{H} & -12.7352 & -42.2099 & 15.4686 \\ \mathrm{H} & -12.8297 & -41.439 & 17.0883 \\ \mathrm{H} & -8.8728 & -45.2898 & 11.4594 \\ \mathrm{H} & -15.1263 & -42.0381 & 16.2659 \\ \mathrm{H} & -12.3373 & -37.8883 & 15.7084 \\ \mathrm{H} & -12.589 & -38.8019 & 17.234 \\ \mathrm{H} & -3.2408 & -27.8466 & 14.2475 \\ \mathrm{H} & -2.0529 & -28.4485 & 15.4699 \\ \mathrm{H} & -0.9813 & -26.7669 & 13.9775 \\ \mathrm{H} & -2.2904 & -25.5819 & 14.3153 \\ \mathrm{H} & -14.6079 & -38.5089 & 14.8967 \\ \mathrm{H} & -14.7284 & -37.7074 & 16.5016 \\ \mathrm{H} & -9.5678 & -44.2494 & 13.7806 \\ \mathrm{C} & -7.4145 & -32.3613 & 11.7744 \\ \mathrm{~N} & -6.3584 & -32.508 & 10.9755 \\ \mathrm{C} & -6.0567 & -33.6783 & 10.4203 \\ \mathrm{~N} & 3.5879 & -30.478 & 9.1663 \\ \mathrm{H} & -6.8619 & -34.714 & 10.6546 \\ \mathrm{H} & -6.8596 & -27.3914 & 20.4668 \\ \mathrm{C} & -7.9448 & -34.5887 & 11.4265 \\ \mathrm{~N} & -8.2089 & -33.4102 & 12.0004 \\ \mathrm{H} & -16.2008 & -39.7549 & 16.37 \\ \mathrm{H} & -14.933 & -39.9399 & 17.6278 \\ \mathrm{~N} & -5.0024 & -33.7869 & 9.7322 \\ \mathrm{~N} & -2.2729 & -34.2227 & 9.9039 \\ \mathrm{C} & -4.5128 & -35.1186 & 9.3012 \\ \mathrm{H} & -4.3371 & -35.0683 & 8.2012 \\ \mathrm{H} & -5.2459 & -35.9364 & 9.4889 \\ \mathrm{H} & -3.1896 & -35.3896 & 10.0453 \\ \mathrm{H} & -2.7478 & -36.3449 & 9.6804 \\ \mathrm{H} & -3971 & -35.507 & 11.136 \\ \mathrm{H} & -32.8721 & 10.25 \\ \mathrm{H} & -32.8721 & 11.3497 \\ \mathrm{H} & -32.0594 & 10.0384 \\ \mathrm{H} & -3195 & 9.5099 \\ \mathrm{H} & -3.4116\end{array}$




$$
\begin{array}{llll}
\mathrm{C} & -9.0193 & -46.3414 & 7.5601 \\
\mathrm{C} & -8.907 & -46.3679 & 6.0282 \\
\mathrm{~N} & -2.3797 & -45.3664 & 8.3038 \\
\mathrm{H} & -7.3731 & -44.8303 & 5.8771 \\
\mathrm{~N} & -6.6045 & -46.8002 & 7.6776 \\
\mathrm{H} & -7.6584 & -46.298 & 14.0506 \\
\mathrm{C} & -5.6503 & -45.5988 & 14.5812 \\
\mathrm{~N} & -0.361 & -43.7297 & 7.1654 \\
\mathrm{C} & -7.518 & -45.8946 & 5.5731 \\
\mathrm{C} & -1.2802 & -44.893 & 9.1812 \\
\mathrm{H} & -0.502 & -45.6916 & 9.1882 \\
\mathrm{H} & -1.609 & -44.7263 & 10.2328 \\
\mathrm{C} & -0.6962 & -43.59 & 8.6062 \\
\mathrm{H} & 0.2177 & -43.3014 & 9.1783 \\
\mathrm{H} & -1.4639 & -42.785 & 8.6976 \\
\mathrm{C} & -6.2616 & -46.8173 & 12.4492 \\
\mathrm{C} & -6.756 & -45.8705 & 13.5509 \\
\mathrm{C} & -0.7726 & -44.9492 & 6.4272 \\
\mathrm{H} & -0.7048 & -44.8219 & 5.3219 \\
\mathrm{H} & -0.0679 & -45.7613 & 6.7227 \\
\mathrm{C} & -2.2124 & -45.3262 & 6.8276 \\
\mathrm{H} & -2.9082 & -44.548 & 6.4329 \\
\mathrm{C} & 5.7374 & -48.4233 & -1.4866 \\
\mathrm{C} & -3.887 & -45.9663 & 12.8061 \\
\mathrm{C} & -4.4024 & -45.0186 & 13.8984 \\
\mathrm{H} & -2.4791 & -46.3187 & 6.3928 \\
\mathrm{C} & 10.0201 & -41.9971 & 0.1179 \\
\mathrm{C} & 10.0942 & -40.5933 & 0.7367 \\
\mathrm{~N} & 3.3594 & -42.5031 & 1.5642 \\
\mathrm{C} & -6.416 & -46.754 & 6.2078 \\
\mathrm{H} & -6.4924 & -47.8039 & 5.8398 \\
\mathrm{H} & -5.4158 & -46.3683 & 5.9041 \\
\mathrm{C} & -7.9278 & -47.2061 & 8.207 \\
\mathrm{H} & 8.7537 & -38.9738 & 1.343 \\
\mathrm{H} & -7.9982 & -47.1297 & 9.3163 \\
\mathrm{~N} & 7.8412 & -42.292 & 1.2583 \\
\mathrm{H} & 6.9374 & -46.735 & -2.1576 \\
\mathrm{H} & -8.9089 & -45.2882 & 7.9126
\end{array}
$$




$$
\begin{aligned}
& \begin{array}{llll}
\text { C } & -5.6583 & -46.4959 & 8.4613
\end{array} \\
& \begin{array}{llll}
\mathrm{N} & -4.4774 & -46.105 & 7.9774
\end{array} \\
& \mathrm{H} \quad-9.6948 \quad-45.71645 .5793 \\
& \begin{array}{llll}
\text { C } & -3.4793 & -45.7573 & 8.7921
\end{array} \\
& \begin{array}{llll}
\mathrm{N} & 1.4659 & -41.8847 & 3.4687
\end{array} \\
& \begin{array}{llll}
\mathrm{N} & -3.6683 & -45.8207 & 10.1114
\end{array} \\
& \begin{array}{lllll}
\text { C } & -4.8315 & -46.2279 & 10.6251
\end{array} \\
& \begin{array}{llll}
\mathrm{N} & -4.99 & -46.3132 & 11.8783
\end{array} \\
& \begin{array}{llll}
\mathrm{N} & -5.8169 & -46.5554 & 9.7857
\end{array} \\
& \begin{array}{llll}
\text { C } & 8.6895 & -39.9929 & 0.8911
\end{array} \\
& \text { H } \quad-10.0287-46.69627 .8793 \\
& \text { C } \quad 2.0593 \quad-43.20591 .4318 \\
& \begin{array}{llll}
\mathrm{H} & 2.1599 & -44.247 & 1.0477
\end{array} \\
& \text { H } \quad 1.4744 \quad-42.6356 \quad 0.673 \\
& \text { C } \quad 1.3211 \quad-43.19582 .7836 \\
& \text { H } \quad 1.778 \quad-43.98243 .4305 \\
& \begin{array}{llll}
\mathrm{H} & 0.2367 & -43.4149 & 2.6379
\end{array} \\
& \begin{array}{llll}
\text { C } & 6.9993 & -46.8273 & 0.0133
\end{array} \\
& \begin{array}{llll}
\text { C } & 6.9694 & -47.5139 & -1.3588
\end{array} \\
& \begin{array}{llll}
\text { C } & 2.0415 & -40.7319 & 2.7327
\end{array} \\
& \text { H } \quad 1.3142 \quad-40.48931 .9231 \\
& \text { H } \quad 2.1443 \quad-39.81513 .3581 \\
& \begin{array}{llll}
\text { C } & 3.3961 & -41.1258 & 2.12
\end{array} \\
& \text { H } 3.6827 \quad-40.41291 .3111 \\
& \text { H } \quad-9.0886 \quad-47.405 \quad 5.6585 \\
& \text { H } \quad-7.4421 \quad-45.935 \quad 4.4599 \\
& \mathrm{H} \quad-6.0228 \quad-44.8879 \quad 15.3575 \\
& \begin{array}{llll}
\text { C } & 4.4587 & -46.9452 & 0.1204
\end{array} \\
& \text { C } \quad 4.441 \quad-47.6325-1.2522 \\
& \text { H } \quad 4.1561 \quad-41.09012 .9338 \\
& \begin{array}{llll}
\text { C } & 7.8058 & -40.8973 & 1.7597
\end{array} \\
& \text { H } \quad 8.2024 \quad-40.92162 .8 \\
& \text { H } \quad 6.776 \quad-40.4747 \quad 1.8071 \\
& \begin{array}{llll}
\text { C } & 9.1523 & -42.9235 & 0.9795
\end{array} \\
& \begin{array}{lllll}
\mathrm{H} & 9.0615 & -43.9188 & 0.4873
\end{array} \\
& \begin{array}{llll}
\mathrm{H} & 11.0459 & -42.4251 & 0.0113
\end{array} \\
& \begin{array}{llll}
\text { C } & 6.7703 & -42.9542 & 1.1319
\end{array} \\
& \begin{array}{llll}
\text { N } & 5.5918 & -42.3913 & 1.4027
\end{array}
\end{aligned}
$$




$$
\begin{aligned}
& \begin{array}{llll}
\text { C } & 4.4511 & -43.0712 & 1.2735
\end{array} \\
& \mathrm{H} \quad-7.0596 \quad-46.953311 .6842 \\
& \text { H } \quad-6.0429 \quad-47.8202 \quad 12.8849 \\
& \mathrm{H} \quad-5.3847 \quad-46.548 \quad 15.1043 \\
& \text { H } \quad-7.0609 \quad-44.903413 .0891 \\
& \begin{array}{llll}
\mathrm{N} & 4.4947 & -44.3404 & 0.8652
\end{array} \\
& \begin{array}{llll}
\text { C } & 5.6618 & -44.9365 & 0.6077
\end{array} \\
& \begin{array}{llll}
\mathrm{N} & 5.703 & -46.1528 & 0.2599
\end{array} \\
& \begin{array}{llll}
\mathrm{N} & 6.787 & -44.2304 & 0.7419
\end{array} \\
& \text { H } \quad 9.5808 \quad-41.9275-0.9057 \\
& \mathrm{H} \quad 10.725 \quad-39.92860 .0989 \\
& \text { H } \quad 8.2231 \quad-39.8812-0.1167 \\
& \begin{array}{llll}
\text { H } & 7.8728 & -46.1384 & 0.0723
\end{array} \\
& \text { H } 7.1219 \quad-47.59050 .8169 \\
& \text { H } \quad 10.5829-40.6513 \quad 1.7375 \\
& \text { H } \quad 7.9036 \quad-48.1064-1.5101 \\
& \begin{array}{llll}
\mathrm{H} & 3.5345 & -46.3361 & 0.2473
\end{array} \\
& \text { H } \quad 4.4694 \quad-47.71050 .9315 \\
& \mathrm{H} \quad-3.0143 \quad-45.5015 \quad 12.2927 \\
& \mathrm{H} \quad-3.5384 \quad-46.921 \quad 13.2649 \\
& \mathrm{H} \quad-3.6011 \quad-44.833514 .6536 \\
& \mathrm{H} \quad-4.6584 \quad-44.033913 .4389 \\
& \text { H } \quad 4.3347 \quad-46.8571-2.048 \\
& \begin{array}{llll}
\mathrm{H} & 3.5569 & -48.31 & -1.3292
\end{array} \\
& \begin{array}{llll}
\mathrm{H} & 9.6491 & -43.0787 & 1.9621
\end{array} \\
& \begin{array}{llll}
\text { C } & 0.2206 & -42.7735 & 6.5767
\end{array} \\
& \mathrm{~N} \quad 0.4796 \quad-41.6576 \quad 7.2586 \\
& \text { C } \quad 1.0629 \quad-40.60946 .6765 \\
& \begin{array}{llll}
\mathrm{N} & 1.6999 & -32.4943 & 8.5974
\end{array} \\
& \begin{array}{llll}
\mathrm{N} & 1.3931 & -40.6807 & 5.3852
\end{array} \\
& \text { H } \quad-8.0744 \quad-48.277 \quad 7.9335 \\
& \text { C } \quad 1.1479 \quad-41.79414 .6894 \\
& \begin{array}{llll}
\mathrm{N} & 0.5658 & -42.8366 & 5.29
\end{array} \\
& \text { H } \quad 5.7169 \quad-48.8929-2.4994 \\
& \text { H } \quad 5.8076 \quad-49.252 \quad-0.7424 \\
& \begin{array}{llll}
\mathrm{N} & 1.2785 & -39.5583 & 7.3452
\end{array} \\
& \mathrm{~N} \quad 0.9382 \quad-36.97158 .4464 \\
& \begin{array}{llll}
\text { C } & 1.952 & -38.399 & 6.7054
\end{array}
\end{aligned}
$$




$\begin{array}{llll}\mathrm{H} & 1.2783 & -38.0227 & 5.8994 \\ \mathrm{H} & 2.9175 & -38.7329 & 6.2562 \\ \mathrm{C} & 2.1987 & -37.2601 & 7.718 \\ \mathrm{H} & 2.5909 & -36.3588 & 7.1917 \\ \mathrm{H} & 2.9521 & -37.5783 & 8.4759 \\ \mathrm{C} & 0.0475 & -38.0873 & 8.8583 \\ \mathrm{H} & -0.2853 & -37.9414 & 9.9136 \\ \mathrm{H} & -0.8397 & -38.086 & 8.1809 \\ \mathrm{C} & 0.7732 & -39.4374 & 8.7359 \\ \mathrm{H} & 1.6538 & -39.4757 & 9.4187 \\ \mathrm{H} & 0.0789 & -40.2594 & 9.0259 \\ \mathrm{C} & 0.5827 & -35.7858 & 8.7032 \\ \mathrm{~N} & 1.3712 & -34.732 & 8.4818 \\ \mathrm{C} & 0.9497 & -33.4979 & 8.7768 \\ \mathrm{~N} & -0.284 & -33.3331 & 9.2621 \\ \mathrm{C} & -1.0938 & -34.3725 & 9.4722 \\ \mathrm{~N} & -0.6382 & -35.5947 & 9.2035 \\ \mathrm{C} & 2.2403 & -30.3396 & 9.6885 \\ \mathrm{H} & 1.93 & -29.2679 & 9.6993 \\ \mathrm{H} & 2.2198 & -30.7139 & 10.7401 \\ \mathrm{C} & 1.2195 & -31.1196 & 8.848 \\ \mathrm{H} & 0.2214 & -31.0895 & 9.3423 \\ \mathrm{C} & 3.0814 & -32.649 & 8.1001 \\ \mathrm{H} & 3.4089 & -33.7132 & 8.0488 \\ \mathrm{H} & 3.1032 & -32.2324 & 7.0655 \\ \mathrm{C} & 4.0295 & -31.8528 & 9.0098 \\ \mathrm{H} & 4.0907 & -32.3423 & 10.0115 \\ \mathrm{H} & 5.0426 & -31.8788 & 8.5422 \\ \mathrm{H} & 1.1154 & -30.6394 & 7.8465 \\ \mathrm{C} & 6.6549 & -29.2934 & 10.6731 \\ \mathrm{~N} & 6.3279 & -28.0191 & 10.9245 \\ \mathrm{C} & 5.0986 & -27.5623 & 10.6539 \\ \mathrm{~N} & 4.1988 & -28.3869 & 10.1067 \\ \mathrm{C} & 4.5083 & -29.6533 & 9.8075 \\ \mathrm{~N} & 5.7375 & -30.0838 & 10.1068\end{array}$

